\title{
Radiation treatment inhibits monocyte entry into the optic nerve head and prevents neuronal damage in a mouse model of glaucoma
}

\author{
Gareth R. Howell, ${ }^{1}$ Ileana Soto, ${ }^{1}$ Xianjun Zhu, ${ }^{1}$ Margaret Ryan, ${ }^{1}$ Danilo G. Macalinao, ${ }^{1}$ \\ Gregory L. Sousa, ${ }^{1}$ Lura B. Caddle, ${ }^{1}$ Katharine H. MacNicoll, ${ }^{1}$ Jessica M. Barbay, ${ }^{1}$ \\ Vittorio Porciatti, ${ }^{2}$ Michael G. Anderson, ${ }^{3}$ Richard S. Smith, ${ }^{1}$ Abbot F. Clark, ${ }^{4}$ \\ Richard T. Libby, ${ }^{5}$ and Simon W.M. John ${ }^{1,6}$ \\ ${ }^{1}$ Howard Hughes Medical Institute, The Jackson Laboratory, Bar Harbor, Maine, USA. ${ }^{2}$ Bascom Palmer Eye Institute, \\ University of Miami Miller School of Medicine, Miami, Florida, USA. ${ }^{3}$ Department of Molecular Physiology and Biophysics, The University of lowa, \\ Iowa City, lowa, USA. ${ }^{4}$ Department of Cell Biology and Anatomy, North Texas Eye Research Institute, University of North Texas Health Science Center,

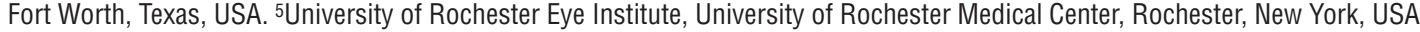 \\ ${ }^{6}$ Department of Ophthalmology, Tufts University School of Medicine, Boston, Massachusetts, USA.
}

\begin{abstract}
Glaucoma is a common ocular disorder that is a leading cause of blindness worldwide. It is characterized by the dysfunction and loss of retinal ganglion cells (RGCs). Although many studies have implicated various molecules in glaucoma, no mechanism has been shown to be responsible for the earliest detectable damage to RGCs and their axons in the optic nerve. Here, we show that the leukocyte transendothelial migration pathway is activated in the optic nerve head at the earliest stages of disease in an inherited mouse model of glaucoma. This resulted in proinflammatory monocytes entering the optic nerve prior to detectable neuronal damage. A 1-time $\mathrm{x}$-ray treatment prevented monocyte entry and subsequent glaucomatous damage. A single $x$-ray treatment of an individual eye in young mice provided that eye with long-term protection from glaucoma but had no effect on the contralateral eye. Localized radiation treatment prevented detectable neuronal damage and dysfunction in treated eyes, despite the continued presence of other glaucomatous stresses and signaling pathways. Injection of endothelin-2, a damaging mediator produced by the monocytes, into irradiated eyes, combined with the other glaucomatous stresses, restored neural damage with a topography characteristic of glaucoma. Together, these data support a model of glaucomatous damage involving monocyte entry into the optic nerve.
\end{abstract}

\section{Introduction}

Glaucoma is a leading cause of visual loss. It is a complex disease that is characterized by the loss of retinal ganglion cells (RGCs) and their axons. Major risk factors include older age and higher intraocular pressure (IOP) (reviewed in ref. 1). Current treatments act to lower IOP but are not effective in many cases (1). Although glaucoma is estimated to affect over 60 million people, more work is needed to understand the molecular mechanisms that damage RGCs (2).

The earliest processes that are necessary for RGC injury in the optic nerve and retina are not defined. No neuroprotective treatments that directly target pathogenic mechanisms in the retina or optic nerve are approved for clinical use against glaucoma. To rationally improve patient care, it is necessary to better understand the etiology and pathogenesis of glaucoma and to develop therapies that target the processes that mediate and/or propagate the initial damage to RGCs and their axons in the optic nerve. Since the injury and demise of RGCs occurs asynchronously, with disease progression typically occurring over decades, there is ample opportunity to target these processes. Targeting the initial damaging mechanisms may be able to prevent glaucoma in some, if not many, individuals with high IOP and would have potential to transform patient care.

Conflict of interest: The authors have declared that no conflict of interest exists. Citation for this article: JClin Invest. 2012;122(4):1246-1261. doi:10.1172/JCI61135.
Studies in humans and animal models have shown that the optic nerve head $(\mathrm{ONH})$ is an important site for glaucoma. RGC axons exit the eye through a specialized region of the ONH that, depending on species-specific anatomy, is known as the lamina cribrosa (LC) or glial lamina. In the LC, bundles of RGC axons pass through astrocyte-lined pores that run through a series of connective tissue beams, which themselves are covered by a network of astrocytes (3). In the glial lamina of rats and mice, the astrocyte network is present, and bundles of axons run through astrocytic pores or glial tubes, but the connective tissue beams are absent (4-6). Despite this difference, the earliest glaucomatous damage to RGCs occurs in this region of the ONH in both species with a LC or glial lamina. Additionally, the characteristic pattern of RGC dysfunction/loss in glaucoma is best explained by the critical requirement for damage to axon bundles in the lamina in both eyes with a LC or glial lamina $(4,6-15)$. RGC axons were shown to be insulted in this region of the $\mathrm{ONH}$ in $\mathrm{DBA} / 2 \mathrm{~J}$ mice, a widely used and inherited glaucoma model (4). Our studies, to understand early damaging processes that are critical for the manifestation of glaucoma, focus on the laminar region of the $\mathrm{ONH}$.

We previously reported that a single dose of $\gamma$-radiation robustly protected the vast majority of treated DBA/2J mice from glaucoma, but we provided no mechanistic insight (16). The magnitude of the treatment effect and its duration remains unprecedented (96\% of treated eyes had no glaucoma a year after treatment; ref. 16). In an 
epidemiological study of 10,000 human atomic bomb survivors in Japan, higher radiation exposure was associated with lower incidence of glaucoma (17). The most common form of glaucoma in Japan does not involve high IOP. Additionally, IOP was elevated in irradiated mice and was unaltered by the radiation treatment. Thus, radiation appears to protect the optic nerve and retina from glaucoma in multiple species, but the mechanisms of protection are not known. Since the radiation treatment completely prevents glaucoma, it is likely to abrogate a critical damaging mechanism in the ONH.

Here, we used a specifically designed gene expression study to identify very early changes that occur in the $\mathrm{ONH}$ during the onset of glaucoma in DBA/2J mice. This genome-wide study found that the first pathway to change significantly is the leukocyte transendothelial migration pathway that is controlled by endothelial cells. Consistent with an early and critical role of this pathway in glaucoma, we show that damaging monocytes enter the ONH of DBA/2J mice prior to neuronal damage but are prevented from entering eyes by the radiation treatment. We also demonstrate that administration of a therapeutically relevant $x$-ray treatment to a single eye is as protective against glaucoma as whole body $\gamma$-radiation.

\section{Results}

Bone marrow transfer is not required for protection. In our previously published study, $\gamma$-radiation was applied to the whole body and head of glaucoma-prone DBA/2J mice at a young age ( $\sim 2$ months), and bone marrow transfer (BMT) was used to allow the animals to survive (16). Administration of precursor cells isolated from bone marrow can protect from retinal degenerations (18), raising the possibility that cells derived from the infused bone marrow conferred the protection against glaucoma. However, given that the atomic bomb survivors did not receive BMT, we reasoned that BMT may not be necessary for the protection from glaucoma in DBA/2J mice. Thus, we tested whether sublethal doses of $\gamma$-radiation, which do not require BMT, protect the mice from glaucoma. Sublethal doses of 5.0 and 7.5 Gy (given at 2 months) were highly protective, with 7.5 Gy protecting as well as the original $10 \mathrm{~Gy}$ plus BMT (Figure 1A). The radiation treatment had no effect on IOP (Supplemental Figure 1; supplemental material available online with this article; doi:10.1172/JCI61135DS1). The protection was long lasting, possibly lifelong (Figure 1A). Thus, the radiation treatment itself and not cells derived from the BMT confers the protection. These lower and therapeutically more relevant doses did not induce cataracts (see Methods), and no long-term damage to any ocular structure was evident.

Radiation protects from early signs of glaucomatous $R G C$ damage. The radiation treatment prevents the loss of RGCs and their axons (16). We used 7.5 Gy $\gamma$-irradiation to determine whether radiation prevents very early glaucomatous damage to RGCs that occurs as a result of IOP elevation. Small and localized axonal swellings, apparent on individual axons in the glial lamina, are among the very earliest morphologically detectable signs of damage in DBA/2J glaucoma (4). They are present before detectable RGC or axon loss. Additionally, dystrophic neurites are detected in the glial lamina at the same early stages of glaucoma (4). The numbers of axonal swellings and dystrophic neurites in the glial lamina of irradiated mice were greatly reduced compared with those in untreated DBA/2J mice (Figure 1, B-D).

Next, we used 7.5 Gy $\gamma$-irradiation to determine whether radiation prevents very early RGC dysfunction. Axonal dysfunction is evident as anterograde transport defects very early in glaucoma (19-21) and was assessed in irradiated and nonirradiated mice using a fluorescent tracer. Anterograde axon transport was completely preserved in almost all irradiated mice but absent in most untreated mice (Figure 1, E and F). The pattern electroretinogram (PERG; ref. 22) is a measure of RGC function, independent of intact axon function, and is greatly reduced in glaucomatous eyes prior to significant RGC loss $(4,23)$. In irradiated mice, PERG remained normal (Figure $1 \mathrm{G})$. Together, our experiments show that sublethal radiation protects RGCs from early glaucomatous damage and dysfunction.

Localized irradiation of a single eye prevents glancoma. Sublethal radiation delivered to the whole animal impacts all tissues/cell types, both within the eye as well as throughout the body. To narrow possible mechanisms (16) and sites that must be irradiated to produce protection, we irradiated either the head or the body (using lead shielding and $10 \mathrm{~Gy} \gamma$-radiation at 2 months). Mice that received head-only radiation were protected from glaucoma, but those that received body-only radiation were not protected (Figure 2, A and B). To assess whether irradiation of just the eyes was protective, we used $x$-ray radiation. Due to the scattering properties and energy of $\gamma$-radiation, it is not possible to irradiate just the eye and shield deeper tissues or the rest of the head from radiation exposure. Like $\gamma$-radiation, $\mathrm{x}$-rays are a form of ionizing radiation, but, unlike $\gamma$-radiation, they can be focused into a beam. Thus, we collaborated with $\mathrm{x}$-ray physicists to build an $\mathrm{x}$-ray machine that produces a 3-mm diameter beam (approximately equal to the length mouse eye) to irradiate the mouse eye (Supplemental Figure 2 and ref. 24). The machine was calibrated so that the retina and optic nerve received the desired dose of $\mathrm{x}$-ray radiation. X-ray energy (35 keV) that resulted in minimal penetration beyond the eye was used. The bone of the eye socket effectively blocked penetration of these $\mathrm{x}$-rays. Using this machine, we assessed a range of $\mathrm{x}$-ray doses (0.6 Gy to $14 \mathrm{~Gy}$, all at 2 months), irradiating both eyes of an animal with the same dose. Demonstrating that ocular irradiation confers protection, eyes exposed to 3.6 Gy or greater were robustly protected from glaucoma (Figure 2, C-F).

$X$-ray irradiation protects only the treated eye. Although the above experiments collectively suggest that ocular tissues must be irradiated to induce protection, they do not determine whether each eye must be irradiated or whether irradiation of only one eye can confer protection to the other nontreated eye. Therefore, to provide further mechanistic insight into the protection, 1 eye of each mouse was exposed to x-rays (7.2 Gy at 2 months). Importantly, the vast majority of treated eyes were completely protected from glaucomatous RGC loss, whereas the untreated eyes were not protected (Figure 2G). These data suggest that a general radiation-induced immunization, regulatory $\mathrm{T}$ cells, or other circulating factors are not primary mediators of the protection. In agreement with this, adoptive transfers of splenocytes from irradiated mice did not confer protection to nonirradiated mice (data not shown). These experiments clearly demonstrate that an eye must be irradiated to be protected, and they reinforce a primary role of local radiationinduced changes within an eye in this protection.

Identifying early stages of glaucoma. As shown above, the radiation treatment prevents very early features of glaucoma. Thus, to understand the protection, we need to identify very early processes that mediate glaucoma. The earliest signs of glaucomatous damage occur in the $\mathrm{ONH}(11,25)$, in which RGC axons are locally insulted (4). Therefore, to suggest biological processes and cell types that mediate early glaucoma and that may be impacted by the radiation-induced protection, we analyzed genome-wide gene expression 
A

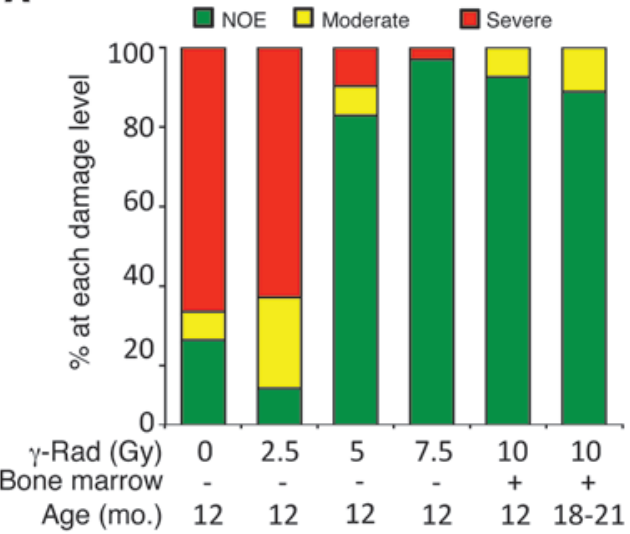

B
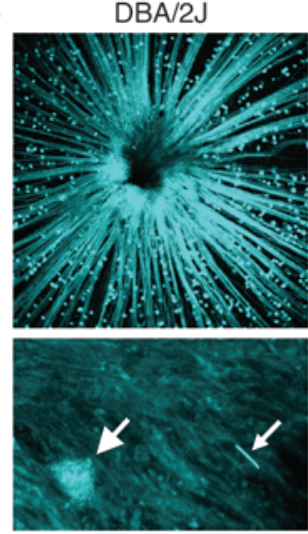

Rad-D2
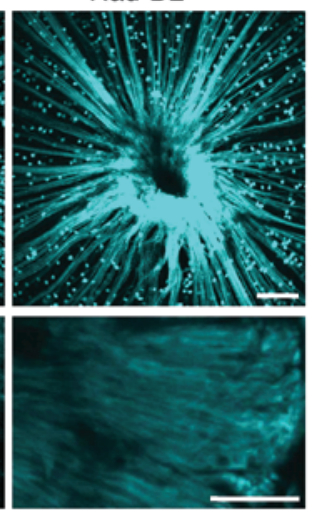

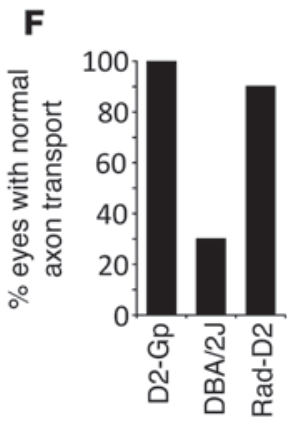

$\mathbf{E}$
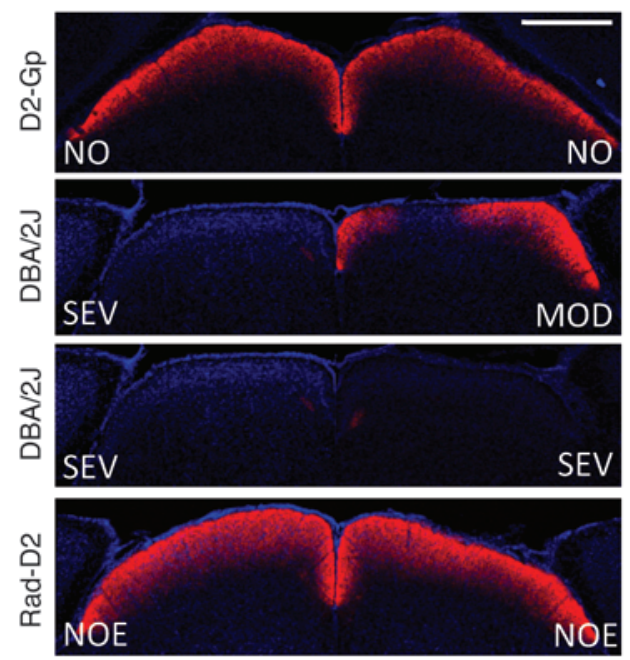

Figure 1

Sublethal $\gamma$-radiation protects DBA/2J mice from glaucoma. (A) Different doses of radiation protect from glaucoma. Most 18- to 21-month-old treated mice had no detectable optic nerve damage (NOE, $n=27)$. Doses of $7.5 \mathrm{~Gy}(n=60,12$ months of age) and $5.0 \mathrm{~Gy}(n=41)$ were highly protective compared to no treatment (0 Gy, $n=140$ ) and equivalent to 10 Gy plus BMT $(n=54)$. 2.5 Gy $(n=35)$ was not protective. (B-D) ONHs of DBA/2J-Thy1 (CFP) and irradiated DBA/2J-Thy1(CFP) 10-month-old mice were assessed for dystrophic neurites (big arrow) and axonal swellings (little arrow) by confocal microscopy. (B) Upper panels show compressed Z stacks of DBA/2J and Rad-D2 eyes imaging from the nerve fiber layer through the glial lamina. (C) Dystophic neurites and (D) axonal swellings were greatly reduced in irradiated compared to untreated eyes ( $n=6$ per group). (E and F) In the majority of 10-month-old Rad-D2 eyes (18/20), anterograde axon transport to the superior colliculus (SC; assessed using a fluorescent tracer) was no different than that of controls (D2-Gpnmb ${ }^{+}$[D2-Gp], 10/10). The majority of untreated DBA/2J mice had severe reductions of labeling in the SC (13/20). The degree of optic nerve damage in corresponding eyes is indicated $(E, N O=$ no damage, MOD = Moderate damage, SEV = Severe damage). (G) In contrast to untreated mice, 12-month-old Rad-D2 mice had normal PERG amplitudes. Untreated had reduced PERG amplitude by 9 months of age ( $n=20$ each group). Scale bars: $100 \mu \mathrm{m}$ (B, top); $20 \mu \mathrm{m}$ (B, bottom); $500 \mu \mathrm{m}$ (E).

patterns in $\mathrm{ONH}$ tissues. Hierarchical clustering is an unbiased computational method for grouping samples based on the similarity of their gene expression profiles. It is a powerful approach for characterizing stages of cancer and other diseases (26-28). Here, we used hierarchical clustering to analyze our gene expression data. We used larger numbers of eyes at early stages of glaucoma than in a previous study (26). We also included eyes that were protected by the radiation treatment. This allowed identification of earlier stages of glaucoma than previously reported. Stage 1 , the earliest reported stage, was subdivided into 3 very early stages (stages $1 \mathrm{a}, 1 \mathrm{~b}$, and $1 \mathrm{c}$; Figure 3). Importantly, the majority of irradiated eyes were most similar to those in stage $1 \mathrm{~b}$ (Figure 3 ). This provides strong molecular evidence that the tissues of radiation-protected eyes experience early glaucomatous stresses, but the molecular pathophysiology is typically stopped at a very early stage.

Glaucomatous stresses/signaling persist in radiation-protected eyes. Pairwise comparisons of stages $1 \mathrm{a}, 1 \mathrm{~b}$, and $1 \mathrm{c}$ and the irradiated DBA/2J group with a strain-matched control group without glaucoma 
A

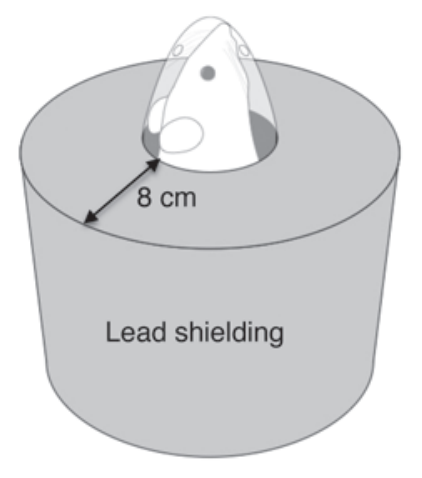

C

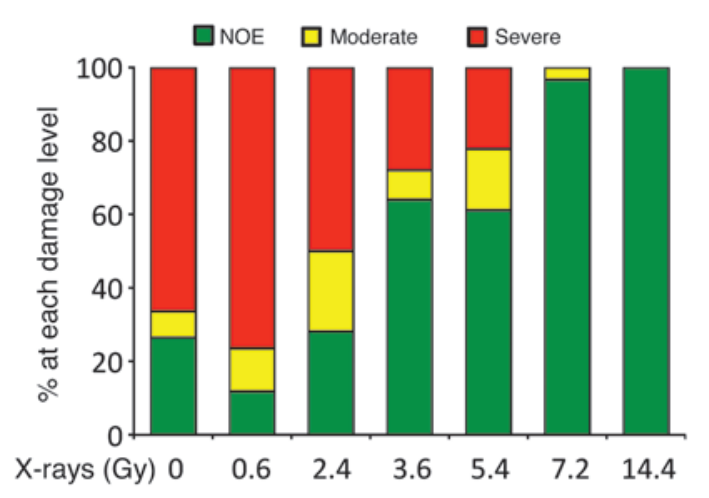

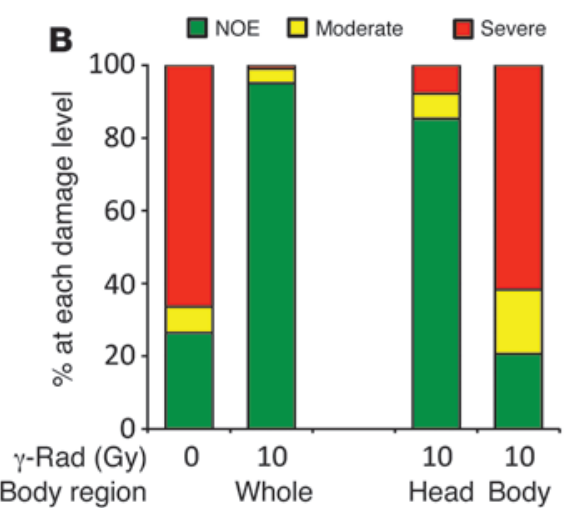

D Optic nerve
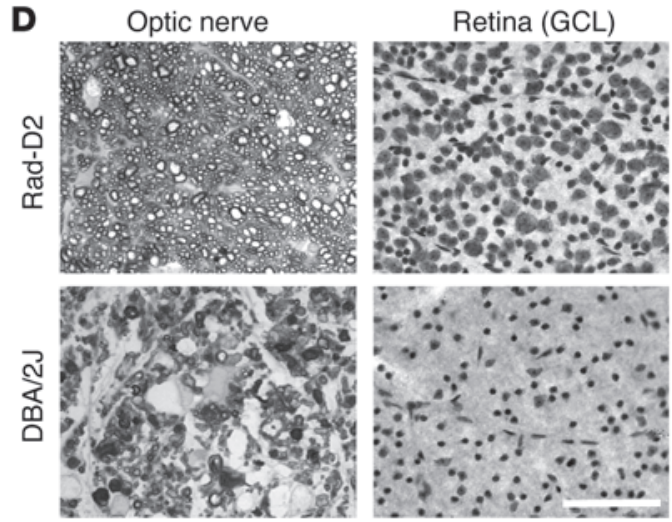

NOE $\square$ Moderate

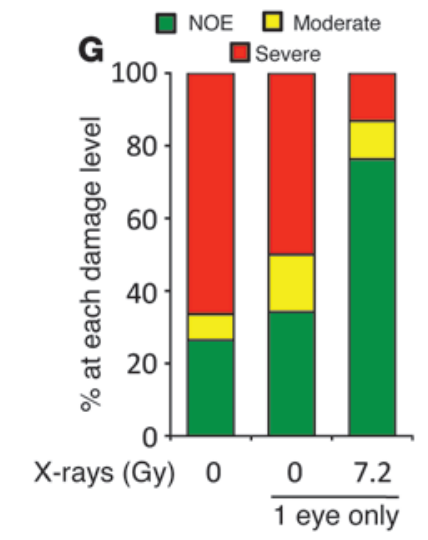

E

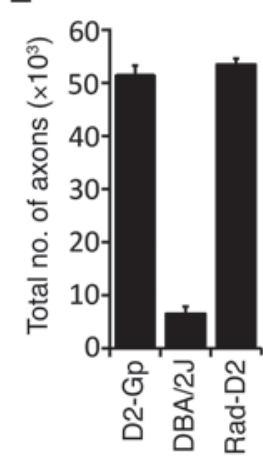

$\mathbf{F}$

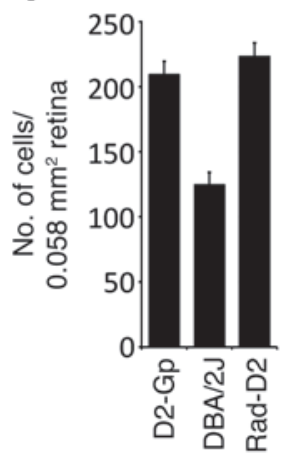

Figure 2

Local radiation robustly protects from glaucoma in DBA/2J mice (assessed at 12 months of age). (A) Lead shielding allowed either head-only irradiation (shown) or body-only irradiation (all of body without head). (B) Irradiation of the head robustly prevented glaucoma compared with untreated eyes ( $P=1.8 \times 10^{-32}$, treated $87 / 102$ eyes, $85 \%$ NOE; untreated $37 / 140$ eyes, $26 \%$ NOE). Body-only irradiation was not protective ( $7 / 34$ eyes, $21 \%$ NOE). For comparison, a summary of the 0 and 10 Gy whole body data from Figure 1 is included. (C-E) X-ray irradiation of just the eye protects from glaucoma. (C) X-ray doses of 7.2 and 14.4 Gy protected the vast majority of eyes from glaucoma (7.2 Gy, $58 / 60$ eyes, $96 \%$ NOE; 14.4 Gy, $40 / 40$ eyes, 100\% NOE). 3.6 Gy and 5.4 Gy more than doubled the number of NOE eyes compared to no treatment (3.6 Gy, $32 / 50$ eyes, $64 \%$ NOE; 5.4 Gy, 33/54 eyes, $61 \%$ ). Doses of 0.6 and 2.4 Gy were not protective (0.6 Gy, $2 / 17$ eyes, $11 \%$ NOE; $2.4 \mathrm{~Gy}, 9 / 32$ eyes, $28 \%$ ). (D-F) Optic nerve and ganglion cell layer (GCL) phenotypes for 12-month-old DBA/2J and radiation-treated (7.2 Gy) DBA/2J mice. Eyes with the most common phenotype for each experimental group were assessed. Optic nerve cross-sections were stained with PPD and flatmounted retinas were stained with cresyl violet. Scale bar: $50 \mu \mathrm{m}$. Protection was evident by $(\mathbf{E})$ axon and $(\mathbf{F})$ ganglion cell layer counts. $(\mathbf{G}) \mathrm{A}$ 1-time $x$-ray dose of 7.2 Gy to a single eye protects from glaucoma, while the untreated fellow eye remained susceptible to glaucoma $(n>40$ for each treatment class, $P=2 \times 10^{-7}$ ).

(D2-Gpnmb $b^{+}$controls) identified differentially expressed (DE; $\left.q<0.05\right)$ genes (Supplemental Figure 3 and Supplemental Tables 1-4). Analysis of DE genes identified 187 probe sets that were DE in each of the stages, $1 \mathrm{a}-1 \mathrm{c}$, and in the irradiated DBA/2J group (Supplemen- tal Table 5). A number of these genes are known to be upregulated by local tissue injury or stress. They encode immunomodulatory, acute phase-response proteins (e.g., Lcn2 and Orm1), complement system proteins (e.g., C3 and C3ar), and chemokine and cytokine/ 


\section{A}

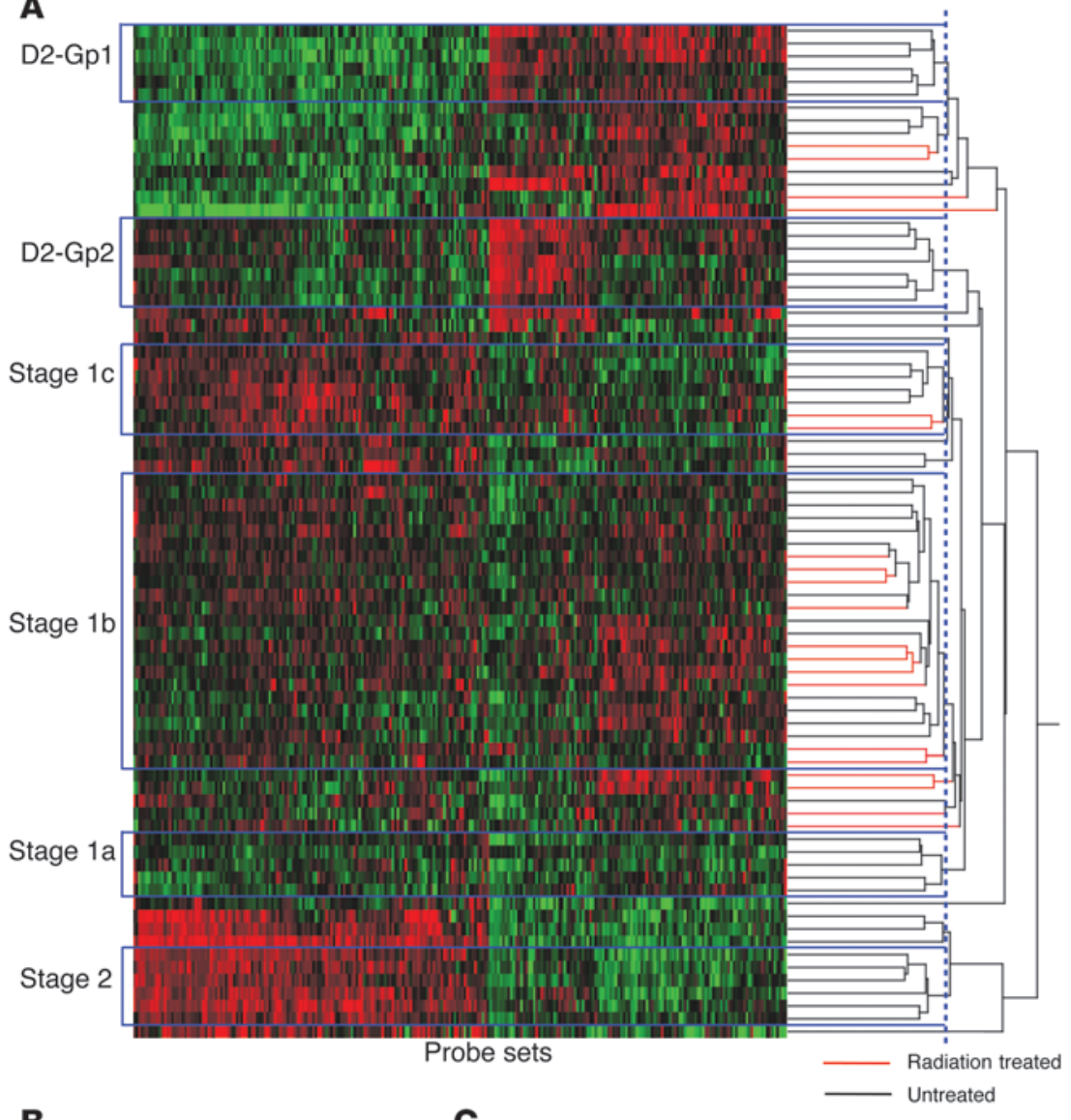

B

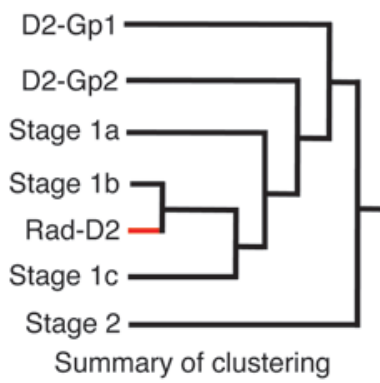

C

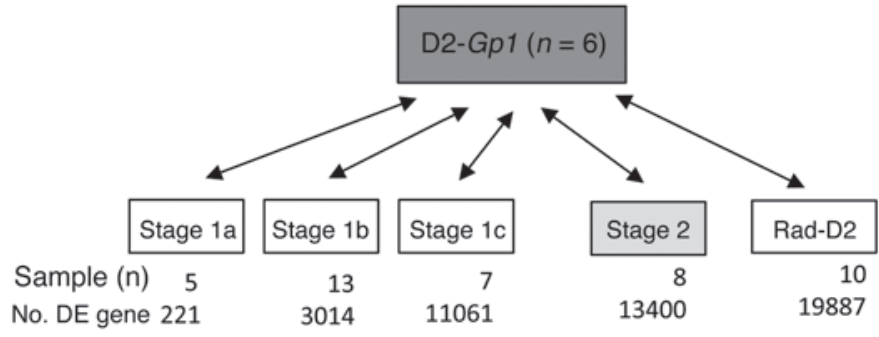

Eyes grouped based on similarity of $\mathrm{ONH}$ expression profiles

Figure 3

Hierarchical clustering identified early stages of glaucoma. (A) Glaucoma-relevant probe sets were used to cluster eyes based on similarity of ONH expression profiles. All eyes included in this study were at early stages, prior to optic nerve damage (see Methods). Four molecularly defined stages containing at least $5 \mathrm{DBA} / 2 \mathrm{~J}$ eyes were identified (stages $1 \mathrm{a}, 1 \mathrm{~b}, 1 \mathrm{c}$, and 2; named for consistency with a previous study, ref. 26). Stages were ordered based on the increasing number of DE genes compared with those in the D2-Gpnmb+ control group (D2-Gp1) and based on the previous study, which also identified stage 2 and subsequent stages. Normalized intensity values for probe sets are represented as "green to black to red," with green indicating lower normalized intensity values, and red indicating higher normalized intensity values. (B) A summary of the relationships among control groups, stages of glaucoma, and the radiation-treated group. The dendrogram shows that radiation-treated eyes (red) are most similar to eyes of stage $1 \mathrm{~b}$. In fact, 10 radiation-treated eyes clustered with the eyes in stage $1 \mathrm{~b}$ (see $\mathbf{A}$ ). This demonstrates that stresses and early molecular changes that occur in glaucoma persist in radiation-treated eyes, but the treatment prevents further progression. The sensitivity of this clustering approach is evident by the splitting of control eyes into 2 groups (D2-Gp1 and D2-Gp2, see Methods). (C) Pairwise comparisons between molecular groups and D2-Gp1. The sample number and the number of DE genes for each group are shown. Similar results were obtained when comparing glaucoma stages to D2-Gp2.

interleukin receptors and antagonists (e.g., Ccrl2, Il6, Il7r, and Illrn). The increased expression of these genes in radiation-treated eyes (Rad-D2), along with the increase in activated microglia inside these eyes (see below), is consistent with the continued exposure of their ONHs to high IOP and glaucomatous stresses.
Early activation ofleukocytetransendothelial migration pathway in glaucoma. Bioinformatics analyses $(29,30)$ of glaucoma stages $1 \mathrm{a}, 1 \mathrm{~b}, 1 \mathrm{c}$, and 2 identified leukocyte transendothelial migration as the earliest process significantly upregulated in untreated DBA/2J eyes compared with that in the controls without glaucoma (Supplemental Figures 


\section{A}

Rad-D2 (vs. Stage 2, q $\leq 0.05)$

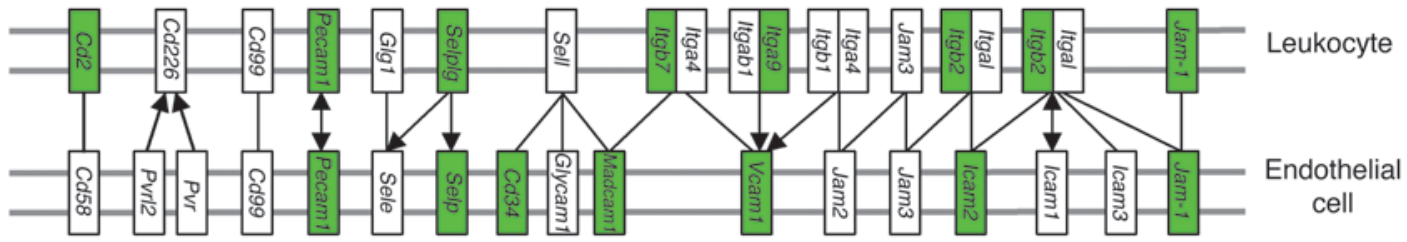

B Rad-D2 (vs. Stage 2, q $\leq 0.05)$

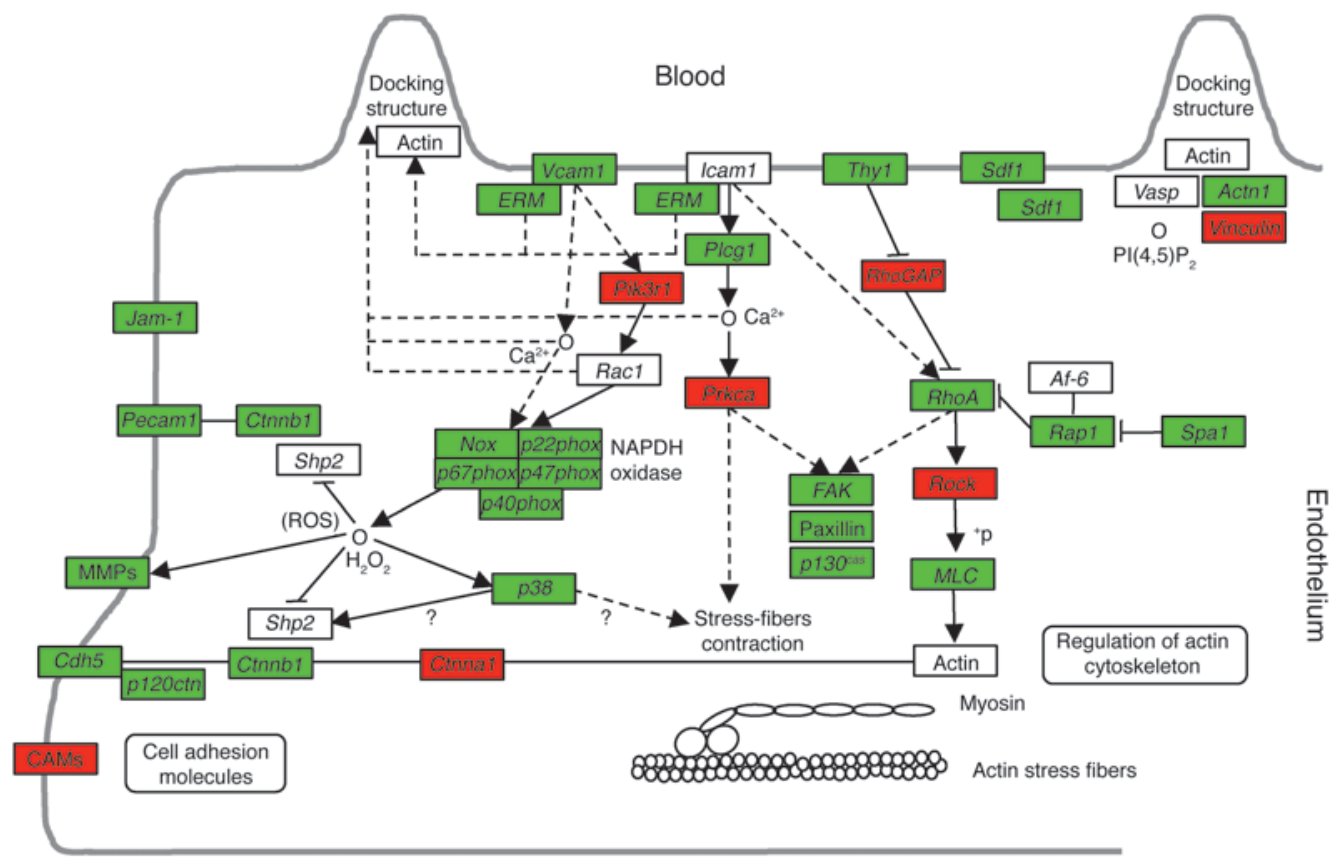

Tissue

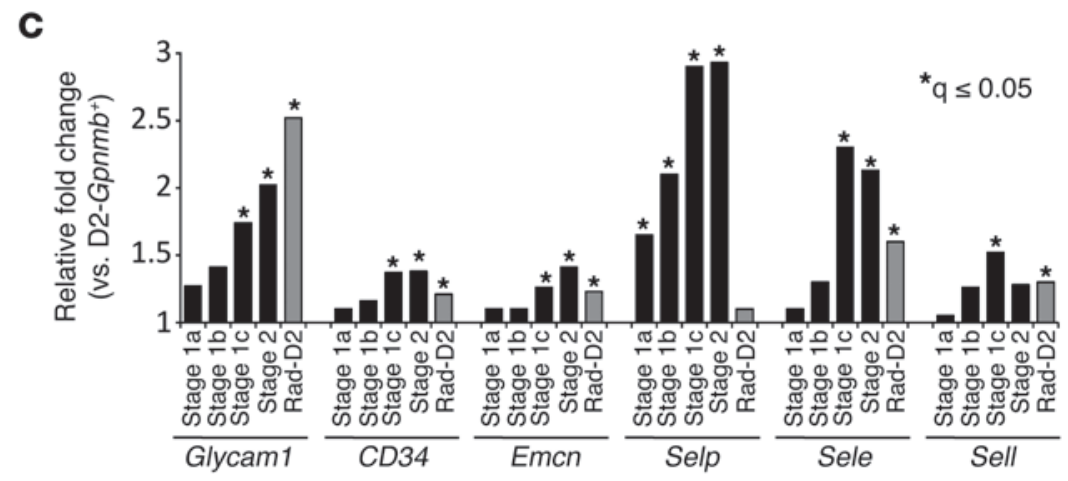

Figure 4

Genes involved in leukocyte transendothelial migration are expressed at lower levels in radiation-treated eyes compared with those in untreated eyes. (A and B) Many genes in the KEGG (A) cell adhesion molecule and (B) leukocyte transendothelial migration pathways are downregulated in the Rad-D2 group compared with those in the glaucoma stage 2 group (green, significantly downregulated; red, significantly upregulated). (C) The expression levels of the major selectins and selectin ligands are generally lower in the radiation-treated group compared with those at glaucoma stages. For instance, $\mathrm{P}$ selectin (Selp) is upregulated by the earliest glaucoma stage but remains indistinguishable from D2-Gpnmb ${ }^{+}$ control values in radiation-protected eyes. In contrast, expression of Glycam1, a glycosylated L selectin ligand, increases in early glaucoma stages and in the radiation-treated group compared with $\mathrm{D} 2-\mathrm{Gpnm} b^{+}$control group. ${ }^{*} q \leq 0.05$.

4 and 5). This is an important finding, which we believe to be novel, which suggests that changes within endothelial cells may be very important early in glaucoma. Leukocyte transendothelial migration is the process by which circulating cells enter tissues from the blood stream in response to inflammatory signaling induced by local tissue stress. Our identification of transendothelial migration as the first pathway to change makes it possible that neuroinflammatory processes play a primary role in initiating glaucomatous damage. 

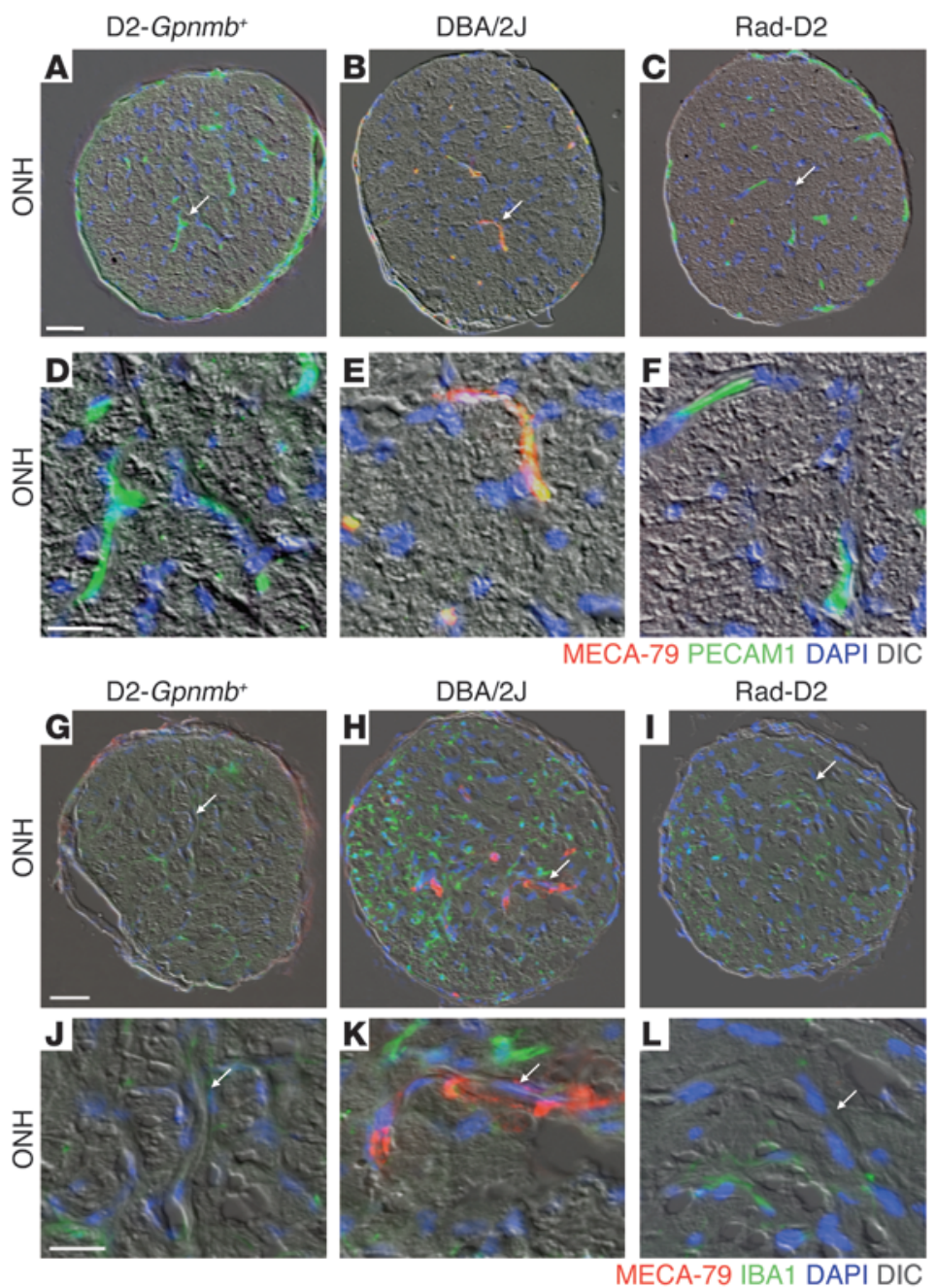

\section{Figure 5}

$\mathrm{L}$ selectin ligands are activated in DBA/2J eyes but not in radiation-treated eyes. The MECA-79 antibody binds to all known, functionally activated $L$ selectin ligands and marks sites of chronic inflammatory signaling. (B, E, H, and $\mathbf{K}$ ) MECA-79 bound to vessels in the ONH of DBA/2J eyes at early stages of glaucoma, prior to detectable optic nerve damage or axon loss. No MECA-79 staining was observed in the ONHs of either $(\mathbf{C}, \mathbf{F}, \mathbf{I}$, and $\mathbf{L})$ radiation-treated or $(\mathbf{A}, \mathbf{D}$, G, and J) D2-Gpnmb+controls. $n=6$ eyes for each group. (H and K) MECA-79 staining coincided with an increase in staining for IBA1, a marker of microglia and monocyte-derived cells. Arrows indicate location of blood vessels, which are shown at higher magnification in the bottom panels (D-F and $\mathbf{J}-\mathbf{L})$. Scale bars: $50 \mu \mathrm{m}$ (A-C and G-I); $20 \mu \mathrm{m}$ (D-F and J-L).

Radiation treatment alters activation of transendothelialmigration pathway. Leukocyte migration into tissues is a highly regulated process, involving interactions between circulating leukocytes and the vascular endothelium. The selectins mediate the first critical step in the leukocyte-endothelial cell adhesion cascade. Consistent with early inflammatory signaling in radiation-protected eyes, many genes in the leukocyte transendothelial migration pathway are DE compared with those in controls. Importantly, many more genes are expressed at significantly lower levels in the irradiated group compared with the glaucoma groups (Figure 4). Overall, the number of DE genes in the leukocyte transendothelial migration pathway is not significantly different between irradiated eyes and control eyes without glaucoma (Supplemental Figure 4). This suggests that the radiation treatment may decrease or prevent cell entry into the eye. Consistent with an effect of the radiation treatment on leukocyte migration into eyes, $\mathrm{P}$ selectin (Selp) gene expression is upregulated by the earliest glaucoma stage but remains indistinguishable from control values in radiation-protected eyes (Figure 4). The expression of other selectin genes is also lower in radiation-protected eyes compared with untreated eyes (Figure 4).

Display of activated L selectin ligands by untreated but not treated eyes. Our data implicate the leukocyte transendothelial migration pathway in both early glaucoma and the protective response to the radiation treatment. To further investigate this, we analyzed L selectin ligands. The display of L selectin ligands by endothelial cells is important for maintenance of a chronic inflammatory response. L selectin-mediated migration of leukocytes into tissue and lymph nodes requires the interaction of $L$ selectin on lymphocytes with peripheral node addressin - a complex of L selectin ligands on endothelial cells (31). Expression of the genes for the glycoprotein ligands of L selectin (glycosylation-dependent cell adhesion molecule 1 [Glycam1], $C d 34$, and endomucin [Emcn]) increased during early glaucoma and in radiation-protected eyes (Figure 4).

To bind L selectin, its ligands require posttranslational modifications, including a critical sulfation $(31,32)$. A sulfation-dependent monoclonal antibody, MECA-79, binds to L selectin ligands at the sulfated epitope that is required for $\mathrm{L}$ selectin binding (31). As local tissue stresses result in cytokine activation of the endothelium and its display of L selectin ligands, MECA-79 is a useful tool for detecting local tissue changes in chronic inflammatory conditions (31). We used MECA-79 to determine the localization of posttranslationally modified L selectin ligands in glaucoma. Importantly, MECA-79 bound to endothelial cells in blood vessels of the optic nerve and retina of DBA/2J mice (Figure 5). During early stages of glaucoma, when optic nerves appear normal and have no axon loss, MECA-79 binding was locally restricted, being absent from the majority of the endothelium in the optic nerve (Figure 5). These data define MECA-79 as an early marker of local glaucomatous changes and implicate L selectin-mediated leukocyte trafficking as an early event in glaucoma. Remarkably, MECA-79 did not bind to endothelial cells of radiation-protected mice, and so these cells lack known, functional $\mathrm{L}$ selectin ligands.

Monocytes enter untreated eyes but rarely enter radiation-treated eyes. The effects of radiation treatment that impair the formation of functional L selectin ligands are expected to influence transendothelial migration and may be a critical component of the protection against glaucoma. To determine whether cells actually cross the endothelium and enter the eye during glaucoma and to assess the influence of radiation, we performed a series of experiments. First, we analyzed the number and morphology of IBA $1^{+}$cells, a marker for monocytes and monocyte-derived cells, by immunofluorescence. Monocytes are recruited to sites of brain injury from the blood and can rapidly differentiate into microglia and other cell types (33). Cell accumulation was greatly reduced in radiation-treated eyes 

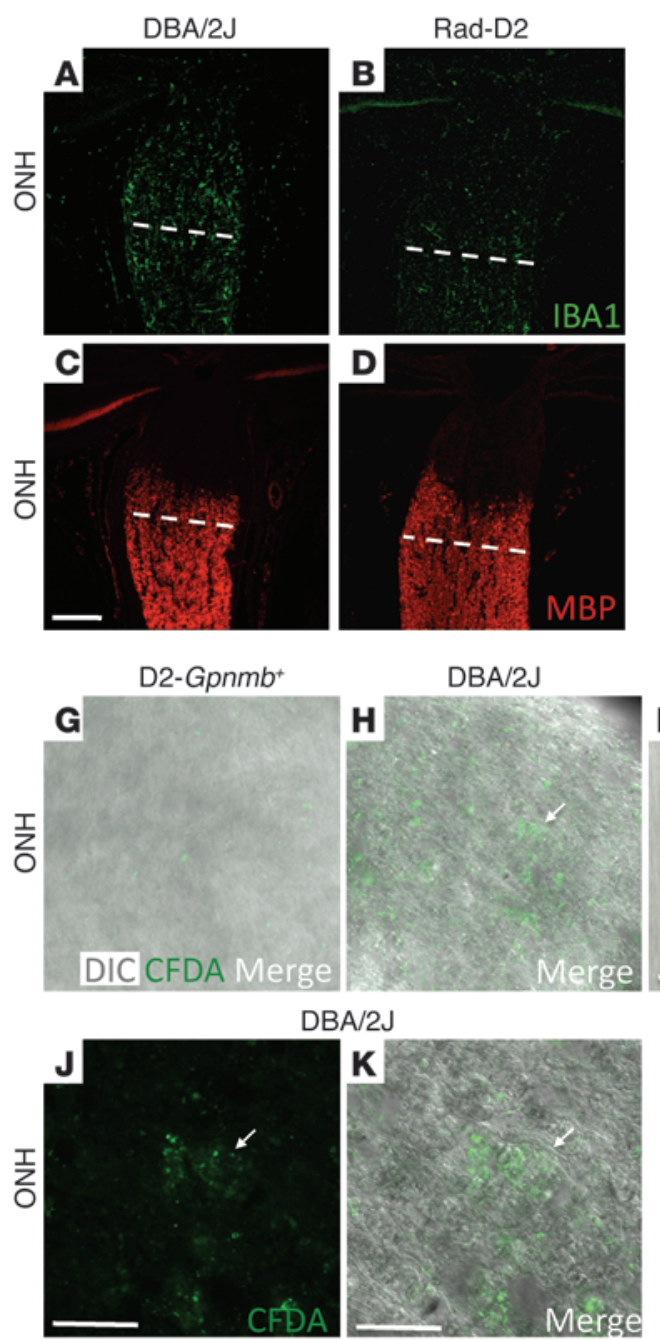

$\mathrm{DBA} / 2 \mathrm{~J}$

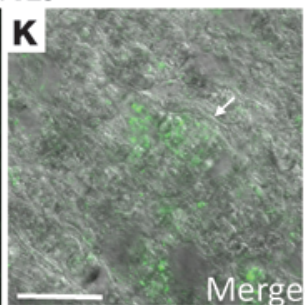

L

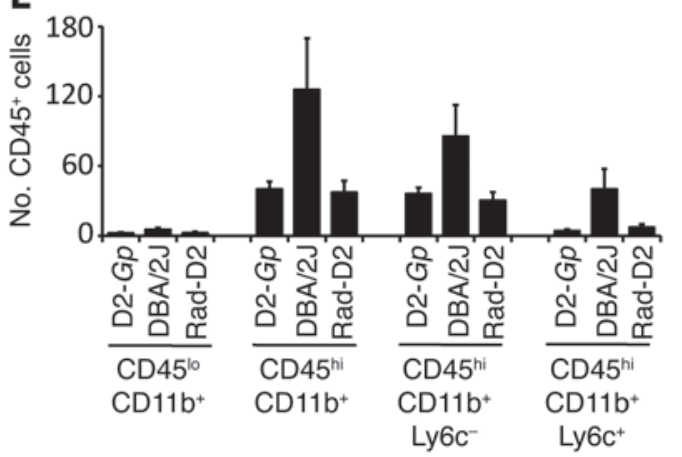

$\mathrm{DBA} / 2 \mathrm{~J}$

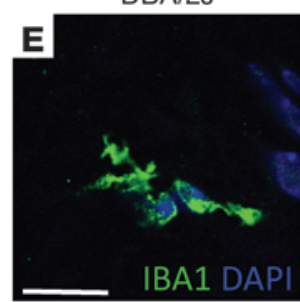

F

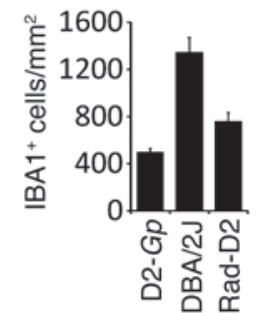

Rad-D2

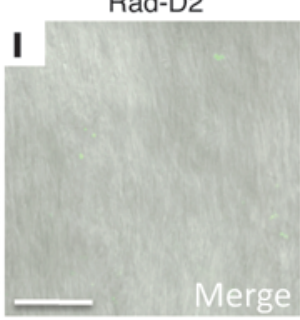

M

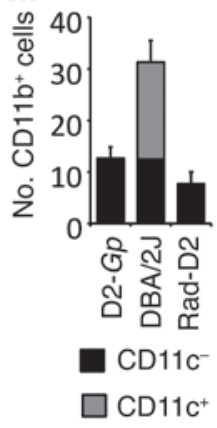

\section{Figure 6}

Cells infiltrate into the ONHs of glaucoma-prone eyes prior to glaucomatous damage but were not detected in radiation-treated eyes. (A-F) IBA1+ cell numbers were assessed from the $\mathrm{ONH}$ to the myelinated region (anterior to white line) of 10.5-month-old DBA/2J, Rad-D2, and D2-Gpnmb $b^{+}$control eyes by immunofluorescence. (E) An example of the counted IBA $1^{+}$cells is shown. The number of IBA $1+$ cells increased in untreated eyes compared with that in controls but there are significantly fewer in Rad-D2 eyes $(P=0.0002)$. $(\mathbf{G}-\mathbf{K})$ Cell infiltration was assessed using CFDA (injected into the spleens; see Methods). $\mathrm{CFDA}^{+}$cells had entered the optic nerves of untreated DBA/2J eyes $(n=8)$ but not Rad-D2 eyes $(n=6)$ or control eyes $(n=6)$. Fluorescent cells must have taken up CFDA in the spleen. $\mathbf{H}$ is a merged view of $\mathbf{J}$ (fluorescent) and $\mathbf{K}$ (DIC). Arrows indicate location of CFDA+ cells. (L) Flow cytometric analysis revealed that CD45hiCD11 b+ monocyte numbers increased in the ONHs of untreated 10.5-month-old DBA/2J eyes compared with those in D2-Gpnmb $b^{+}$controls and radiation-treated eyes $(P=0.01)$. $\mathrm{CD} 45^{\text {hi }} \mathrm{CD} 11 \mathrm{~b}^{+}$monocyte numbers did not increase in radiation-treated eyes compared with $\mathrm{D} 2-\mathrm{Gpnmb} \mathrm{b}^{+}$controls $(P=0.96)$. CD45 hi is a marker of blood-derived infiltrating cells. One-third of the CD45 ${ }^{\text {hi }} \mathrm{CD} 11 \mathrm{~b}{ }^{+}$cells were also positive for the proinflammatory marker Ly6c ${ }^{+}$. (M) Flow cytometric analysis also shows that the CD45i cells that infiltrate into DBA/2J eyes are CD11b+CD11 $\mathrm{c}^{+}$double-positive monocytes. This class of cell is completely absent in radiation-treated eyes $(P=0.0007$, compared with untreated eyes). Scale bars: $50 \mu \mathrm{m}(\mathbf{A}-\mathbf{C}$ and $\mathbf{G}-\mathbf{I})$; $20 \mu \mathrm{m}$ (D and $\mathbf{E}$ and $\mathbf{J}$ and $\mathbf{K}$ ). compared with that in untreated eyes (Figures 6 and 7). In untreated DBA/2J retinas, both ramified and round $\mathrm{IBA} 1^{+}$cells accumulate and are in a responding state (previously called activated; see ref. 34 and Figure 7). In radiation-treated eyes, there was no significant difference in the number of responding ramified cells (likely microglia) compared with that in untreated eyes, but the number of round cells was significantly reduced (Figure 7).

We used the tracer carboxyfluorescein diacetate (CFDA) to directly determine whether cells cross the endothelium and enter the eye during glaucoma. CFDA was injected into the spleens of untreated and irradiated DBA/2J mice to label leukocytes that will enter the circulation. Seventy-two hours later, fluorescently labeled cells were readily detected in the ONHs and retinas of DBA/2J eyes but were not detected in radiation-protected eyes (Figures 6 and 7). This shows that blood-derived cells enter the $\mathrm{ONH}$ during early glaucoma and that this entry is greatly diminished in radiation-protected eyes.

Radiation-treatment prevents entry of monocytes with a damaging marker profile. To further assess cell entry into the eye during glaucoma and the effects of the radiation treatment, we used flow cytometry. Confirming the migration of cells into eyes from the circula- 

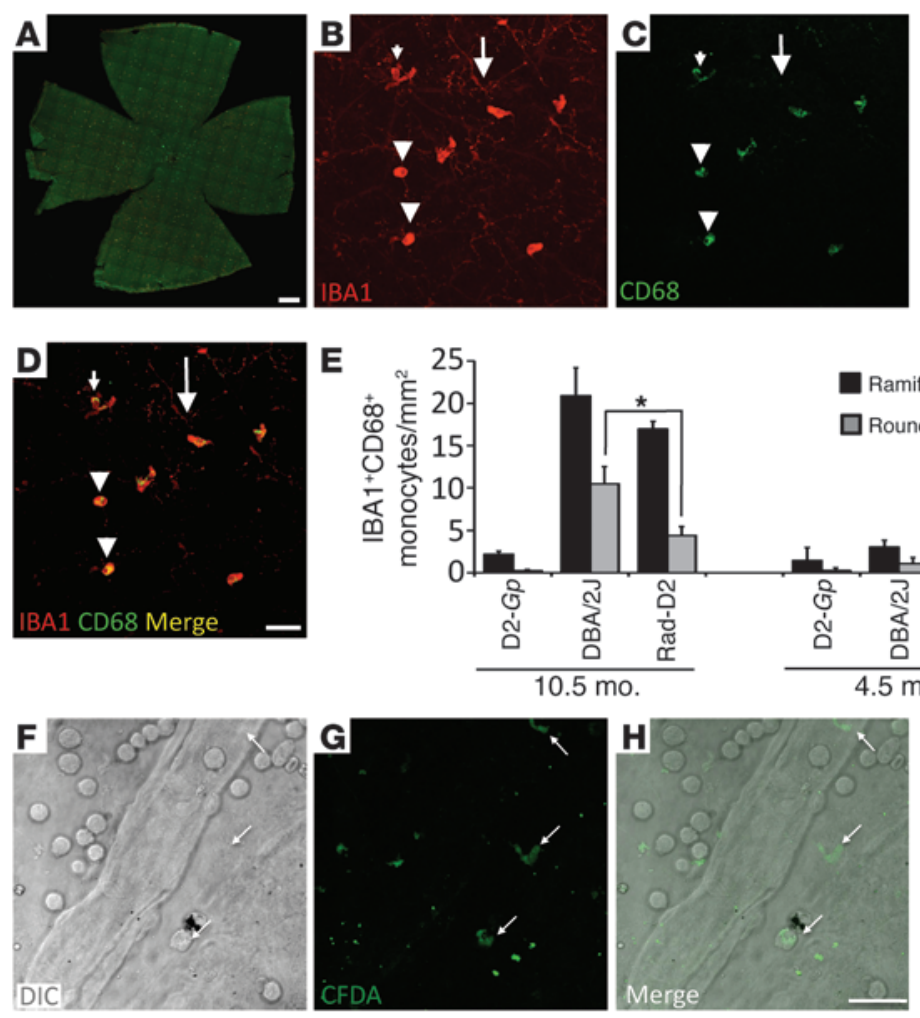

\section{Figure 7}

Entry of cells into the retina from the blood occurs in early glaucoma but is greatly reduced in radiation-treated eyes. (A-D) The entire nerve fiber layer and ganglion cell layer of flat-mounted retinas was assessed for IBA $1{ }^{+}$CD $688^{+}$cells. CD68 is a marker of activated or responding cells (95). Cells were classed as either ramified (elongated bodies and obvious processes) or round (rounded bodies that lacked prominent processes). (B-E) Images from untreated DBA/2J eyes are shown as examples. In untreated DBA/2J and radiation-treated eyes, mosaics of IBA1+CD68- microglia were present (big arrows). In untreated eyes, the number of ramified (small arrows) or round (arrowheads) IBA $1^{+} \mathrm{CD} 68^{+}$cells increased significantly ( $P<0.001$ for both comparisons) compared with that in controls. In radiation-treated eyes, the number of $\mathrm{CD}_{6} 8^{+}$ramified cells also increased significantly compared with that in controls and was not significantly different than that in DBA/2J eyes $(P=0.2)$. However, there were significantly fewer CD68+ round cells in radiation-treated eyes $\left({ }^{*} P<0.001\right)$. No changes were observed to IBA $1^{+}$cells in other layers of the retina (data not shown). ( $\mathbf{F}-\mathbf{H})$ Infiltration of cells was assessed using CFDA in 10.5-month-old mice. Both round and ramified cells that were positive for CFDA (arrows) were present in the retinas of DBA/2J eyes, indicating infiltration from the blood stream. CFDA ${ }^{+}$cells were not detected in the retinas of radiation-treated or D2-Gpnmb+ control eyes (data not shown). Scale bars: $500 \mu \mathrm{m}$ (A); $50 \mu \mathrm{m}$ (B-D); $10 \mu \mathrm{m}$ (F-H). tion, cells with a high expression of CD45 (CD45 hi ; a marker of blood-derived cells) were present in untreated eyes at an early stage of glaucoma (Figure 6, L and M). One-third of CD45 hi cells were positive for the proinflammatory marker Ly6c (35). Importantly, there was no increase in CD45 lo cells (resident cells). The number of CD $45^{\text {hi }}$ cells did not increase in radiation-treated eyes compared with that in controls without glaucoma. Other than microglial and monocyte-derived CD11 $\mathrm{b}^{+}$cells, essentially no other immune cell types, including T- and B-lymphocytes, were detected (Supplemental Figure 6). Again, the number of these CD11 $\mathrm{b}^{+}$cells was much lower in radiation-treated mice compared with that in untreated DBA/2J mice (Figure 6, L and M, and Supplemental Figure 6). In the $\mathrm{ONH}$, the majority of $\mathrm{CD} 11 \mathrm{~b}^{+}$cells were also $\mathrm{CD} 11 \mathrm{c}^{+}$in glaucomatous eyes. $\mathrm{CD} 11 \mathrm{~b}^{+} \mathrm{CD} 11 \mathrm{c}^{+}$cells are likely to have properties of both DCs and macrophages $(36,37)$. Compared with DCs, they can have lower antigen-presenting and higher phagocytic activity $(36,38)$. Infiltrating, $\mathrm{CD} 45^{\mathrm{hi}} \mathrm{CD} 11 \mathrm{~b}^{+} \mathrm{CD} 11 \mathrm{c}^{+}$cells are early responders to experimentally induced optic nerve injury (37) and appear to play a role in the damaging inflammatory response

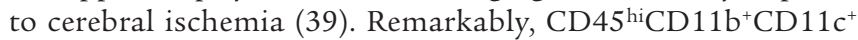
monocytes were absent in radiation-protected eyes (Figure 6M). Since the earliest known insult to RGC axons occurs in the ONH, and because radiation treatment prevents all detectable signs of glaucoma, including functional defects, it is possible that these monocytes enter the eye and initiate RGC injury.

Further experiments support the theory that radiation protects by preventing monocyte entry. If radiation treatment protects from glaucoma by preventing the entry of monocytes into the eye, we can make 2 predictions. First, radiation would not be expected to protect from mechanical insults that directly damage RGCs. To test this, we directly damaged RGCs by crushing their axons in the optic nerve. As predicted, the radiation treatment conferred no protection against this direct optic nerve injury (Supplemental Figure 7). Second, adding damaging mediators that are produced by monocytes that enter untreated eyes, but are reduced or absent in radiation-treated eyes, may induce RGC demise in treated eyes. Endothelin-2 is produced by monocytes that enter untreated eyes. The endothelins are implicated in human glaucoma and a variety of relevant animal models (26, 40-43). Expression of the endothelin-2 gene, $E d n 2$, is significantly decreased in radiation-treated eyes, which also have greatly reduced (almost absent) numbers of EDN2 ${ }^{+}$monocyte-derived cells (Figure 8). Importantly, intravitreal injection of EDN2 induces substantial damage in radiation-treated DBA/2J eyes but not in control mice (Figure 9). This substantial damage is only induced during the period of IOP elevation. This is not unexpected, as other damaging mediators in addition to EDN2 are made by monocytes, and other IOP-induced stresses are expected to conspire with EDN2 to damage the neurons. Importantly, the EDN2-induced damage occurs in sharply delimited sectors (a key hallmark of glaucoma; refs. 6,14 ), and the proportion of eyes with damage matches that of untreated glaucomatous DBA/2J mice. Collectively, our data provide strong evidence that monocytes mediate early damage in glaucoma and that radiation treatment protects from glaucoma by preventing their entry into the eye.

\section{Discussion}

Our data provide several fundamental advances in understanding glaucoma. First, our gene expression data and subsequent functional tests showed that local stress in neural tissues, in response to high IOP, induces proinflammatory signaling and the activation of transendothelial migration. The transendo- 
A

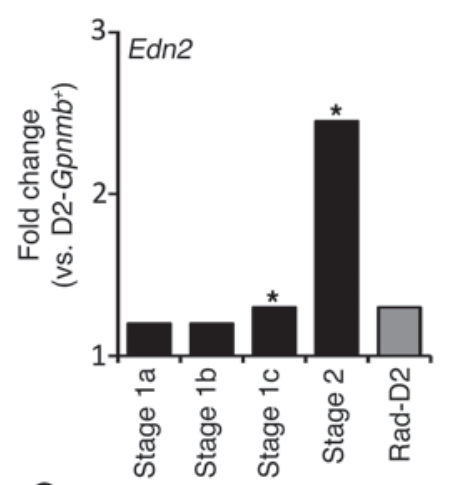

C
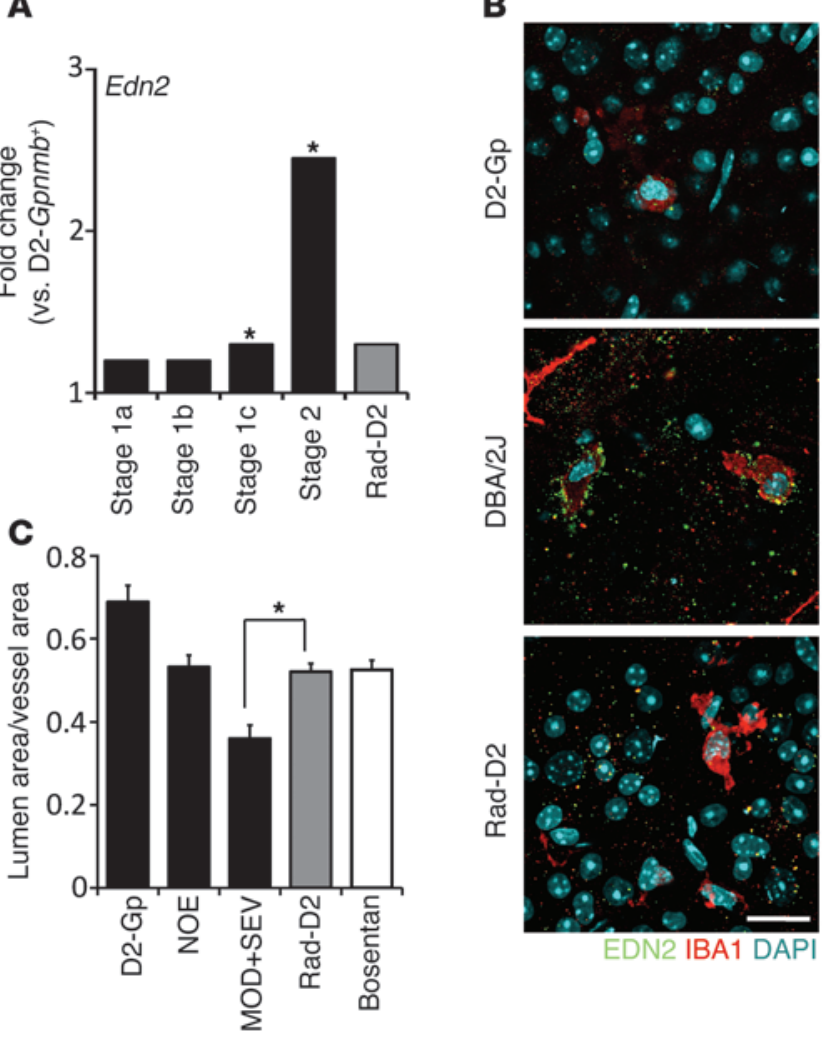

thelial migration pathway is the first pathway to significantly change in glaucoma, being activated prior to changes in highly sensitive measures of neuronal dysfunction and neural damage. Second, we clearly showed that the entry of monocytes (and/or monocyte-derived cells) into the eye occurs very early in DBA/2J glaucoma and prior to neural damage. Third, we showed that a 1 -time treatment with $\mathrm{x}$-rays protects a treated eye from glaucoma. This treatment alters endothelial cell activation in the face of proinflammatory signaling and abrogates migration of monocytes into the eye. Adding a damaging molecule that is made by the monocytes to irradiated eyes, along with other glaucomatous stresses, restores neural damage, with a topography that is characteristic of glaucoma. Together, these data strongly imply that monocyte entry is required for neural damage to occur. However, further experiments that prevent monocyte entry independent of any radiation treatment are required to definitively prove if this is so. Although not directly assessed, a functional role of invading monocytes in glaucomatous RGC death may be supported by an independent study showing that a mutation in the Itgam gene, which encodes CD $11 \mathrm{~b}$, protects from RGC death subsequent to laser-induced IOP elevation (44). Although increased expression of proinflammatory molecules was previously documented in glaucoma and animal models with experimentally raised IOP (26, 45-50), in most studies they were not timed in relation to disease stage, and it was not known that these changes were required for neural damage. In addition to endothelial cells and leukocytes, neuroinflammatory processes are likely to be controlled and mediated by both microglia and astrocytes $(34,51)$ in the $\mathrm{ONH}$ during glaucoma. For example, astrocytes may initially respond to high IOP and/or other glaucomatous stresses by activating

\section{Figure 8}

EDN2 increases in glaucoma but not in radiation-treated eyes. (A) Edn2 expression is upregulated in glaucoma but not in radiation-treated eyes. (B) EDN2+IBA $1{ }^{+}$cells are readily detected in retinas of untreated DBA/2J eyes but are rare in radiation-treated eyes. Scale bar: $20 \mu \mathrm{m}$. (C) Endothelin-2 is a potent vasoconstrictor, and the ratio of vascular lumen to overall vessel diameter was decreased in DBA/2J eyes with moderate and severe optic nerve damage (MOD+SEV) compared with that in eyes with no glaucoma (NOE, $P=0.001$ ). This ratio was not decreased in Rad-D2 eyes compared with that in NOE eyes $(P=0.78, n>6$ in all groups). Rad-D2 eyes had very little EDN2, and vasoconstriction was prevented to the same degree as in eyes treated with an EDN receptor antagonist (Bosentan, ref. 96, $P=0.90)$. ${ }^{*} q \leq 0.05$.

early inflammatory signaling (reviewed in ref. 52). It is likely that early inflammatory signaling protects against local tissue stresses (metabolic/biological stress and mechanical strain) or injury. A protective role of ocular inflammation against RGC degeneration has been shown after axon injury $(53,54)$.

However, after prolonged IOP elevation and/or exposure to other stresses, the initially protective response may develop into chronic and damaging inflammation. Immune dysfunction is reported to contribute to some forms of glaucoma (55), and both protective and damaging roles are suggested for $\mathrm{T}$ cells (reviewed in ref. 56). However, the roles of $\mathrm{T}$ cells in glaucoma require further clarification. In agreement with some published studies (57), our current study did not detect or implicate $\mathrm{T}$ cells. In another study, experimental induction of high IOP upregulated the cytokine TNF. This induction was sequentially followed by microglial activation, loss of oligodendrocytes, and delayed loss of RGCs. It was suggested that microglial activation damages oligodendrocytes, resulting in RGC loss (44). However, oligodendrocyte loss occurs later in the inherited $\mathrm{DBA} / 2 \mathrm{~J}$ glaucoma and appears to be a secondary response to damage (58).

To our knowledge, the present study is the first to show the transendothelial migration of monocytes into neural tissue of the eye after IOP elevation and to suggest a primary importance of this monocyte entry for glaucomatous neural damage. Leukocyte migration into tissues is a highly regulated process, involving complex interactions between circulating leukocytes and the vascular endothelium. The selectins are key adhesion molecules displayed on leukocytes and endothelial cells. They mediate the first critical step in the leukocyte-endothelial cell adhesion cascade and are necessary for leukocyte tethering, rolling, and subsequent transendothelial migration from blood vessels into tissues (reviewed in ref. 59). Unexpectedly, our data implicate the aberrant regulation of selectins and their ligands as an early pathogenic event in endothelial cells of the ONH that is required for the initiation of glaucomatous damage. Also unexpected, our data show that a 1-time radiation treatment prevents the display of activated $\mathrm{L}$ selectin ligands by endothelial cells at a much later age. Endothelial cells in radiation-treated eyes do not display functional $\mathrm{L}$ selectin ligands, as assessed by MECA-79, an antibody that specifically detects functional ligands and serves as a new and early biomarker for glaucoma. Expression of E selectin was previously reported as a biomarker in the drainage structures of human eyes with glaucoma, but it was not determined whether this was an early pathologic event or a secondary response to damage (60). That report did not assess whether E selectin had a pathophysiological role in either IOP elevation or glaucomatous neurodegeneration. 

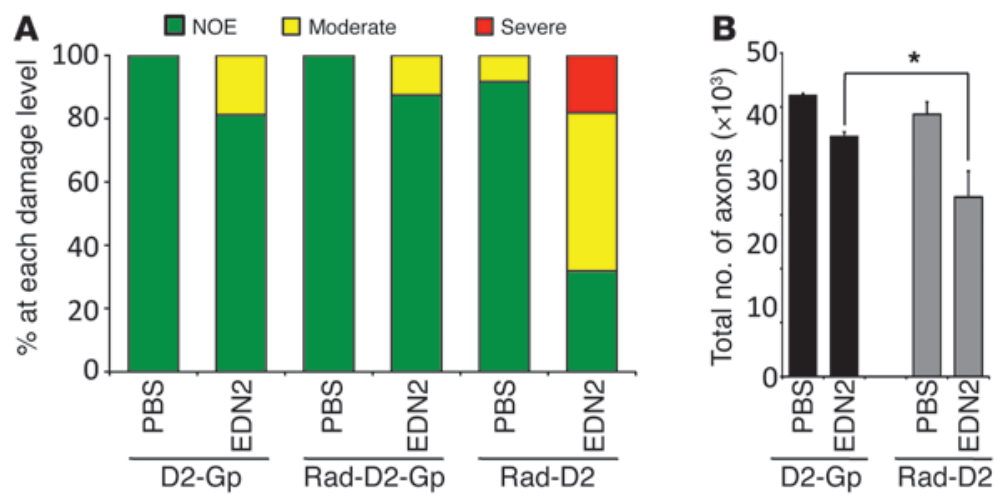

C

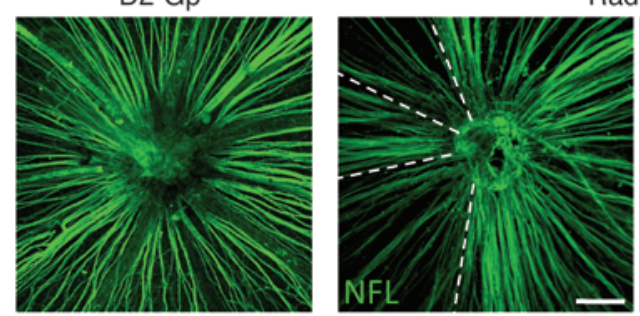

Retina
Rad-D2

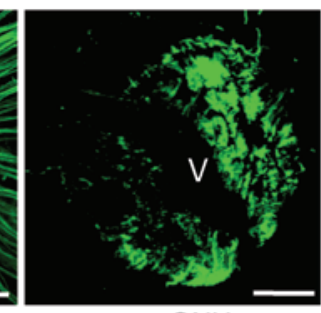

$\mathrm{ONH}$

\section{Figure 9}

Restoring EDN2 to radiation-treated eyes induces a pattern of damage that is characteristic of glaucoma. EDN2, injected into the vitreous of radiation-treated eyes, caused glaucoma-like RGC damage that was not observed in control eyes. (A) Most radiation-treated, EDN2-injected $\mathrm{DBA} / 2 \mathrm{~J}$ eyes had moderate and severe optic nerve damage. This was not the case for EDN2-injected untreated D2-Gp or radiation-treated D2-Gp eyes or eyes injected with vehicle ( $1 \times \mathrm{PBS}, n>15$ each group, all age matched). D2-Gp mice do not develop high IOP and glaucoma, and so EDN2 induces the most damage in eyes that have high IOP and possibly other stresses that are not sufficient by themselves to induce glaucoma. Thus, EDN2 is a damaging factor that conspires with other stresses to induce glaucoma but is diminished in radiation-treated eyes. (B) Axon counts demonstrated a significant increase in axon loss in radiation-treated, EDN2-injected DBA/2J eyes compared with that in D2-Gpnmb ${ }^{+}$controls $\left({ }^{*} P<0.0001\right)$. (C) Radiation-treated, EDN2-injected DBA/2J eyes had fan-shaped patterns of RGC loss, with discrete borders in the retina and regional axon loss in the ONHs (key characteristics of glaucoma). NFL, Anti-neurofilament antibody. Fan-shaped patterns were not observed in D2-Gpnmb ${ }^{+}$ or radiation-treated D2-Gpnmb $b^{+}$controls. V, vessel. Scale bars: $100 \mu \mathrm{m}$ (retina); $50 \mu \mathrm{m}(\mathrm{ONH})$.
It is not simple to envision how radiation protects in the long term, especially as early glaucomatous stresses are still present in radiation-treated eyes. One possibility is that radiation induces long-lasting epigenetic changes (61) that alter gene expression and prevent glaucoma. Given the need to treat local cells for protection, it is likely that the radiation somehow alters a local cell population, or possibly populations of different cell types, that normally contribute to the early pathogenesis of glaucoma. Possibly affected cell types include stem cells, resident microglia, and astrocytes (16). Stem cells have not been implicated in glaucoma pathogenesis, and we have not documented obvious changes in resident microglia in radiation-treated eyes, compared to untreated eyes, at these early stages. Under stress conditions, astrocytes can produce cytokines and other molecules and so are capable of initiating and controlling early inflammatory responses in glaucoma (reviewed in ref. 51). Our gene expression data show that the early inflammatory responses are intact in radiation-treated eyes but that the entry of monocytes is prevented. Collectively, our data strongly support transendothelial migration of monocytes as a primary step for initiating or propagating neural damage during early stages of glaucoma, a step that is prevented by radiation treatment.

It is not clear how radiation suppresses invasion of monocytes in the long term, including still-unborn monocytes that are remotely located. Endothelial cells are a key cell type in locally controlling transendothelial migration of monocytes and the nature of inflammatory responses. Our data show that endothelial cells are somehow changed by the radiation treatment. In radiation-treated eyes, there is altered expression of various endothelial genes that control transendothelial migration. Additionally there is abrogated activation of $\mathrm{L}$ selectin ligands. Thus, endothelial cells may be directly impacted by the effects of irradiation, resulting in modulation of a neuroinflammatory process that is critical for glaucoma development. Expression of the endothelial gene Glycam1 is greater in radiation-protected eyes than in early glaucoma (Figure 4C). GLYCAM1 is one of the glycoprotein ligands for L selectin, which is secreted from endothelial cells and is present in normal serum (62). Expression of Glycam1 is upregulated in 4.5-month-old mice ( 2 months after irradiation; data not shown), long before the onset of glaucoma in our colony of DBA/2J mice. Thus, the steady-state expression of Glycam 1 is increased long term and may explain the radiation protection. Secreted GLYCAM1 is likely to modulate endothelial-leukocyte interactions, leukocyte-leukocyte interactions, and L selectin-mediated intracellular signaling that modulates transendothelial migration (63). GLYCAM1 secretion was inversely related to leukocyte transendothelial migration from blood (64). Thus, the radiation treatment induces a long-term increase in Glycam1 expression, which may be a key factor inhibiting entry of monocytes into the optic nerve during very early glaucoma. Further experiments will test this possibility. If correct, the manipulation of Glycam 1 expression may prove effective against a variety of inflammatory conditions.

Our data support what we believe to be a new model in which monocytes are essential for glaucomatous damage and suggest that glaucoma is primarily a neuroinflammatory disease. Much of the field has focused on astrocytes, microglia, and RGCs themselves, with some interest in the endothelial control of blood flow (65-67). Our demonstration that specific radiation of the eye can prevent glaucoma is an important finding. Since localizing tissue irradiation to the relevant tissue is therapeutically desirable, we developed an $x$-ray machine to specifically irradiate the mouse eye. Ocular radiation proved highly effective at preventing glaucoma, being much more effective than even the most robust of the other reported treatments in the same mouse model (e.g., refs. $26,68-70$ ), with the exception of whole body irradiation (16). It will be important to extensively evaluate the efficacy and safety of this treatment in other mammalian and primate models. The potential dangers of radiation exposure are well documented. Side effects vary depending on the dose and length of exposure but can include cataracts and cancer (71). It is difficult to explain why some individuals are detected with glaucoma subsequent to 
radiation treatment for ocular tumors. The different outcomes may result from parameters that differ to those in our experiments and may include age at treatment, radiation dose or exact timing and duration of treatment, and/or individual genetic makeup. On the other hand, it is possible that radiation treatment (or other treatments that alter transendothelial migration) may have prevented glaucoma in some individuals, but this is not known as it is not possible to predict glaucoma development prior to the onset of disease features. The great success of localized $\mathrm{x}$-ray radiation in our study suggests that it may be possible to develop safe, radiation-based treatments of the eye or optic nerve that may be effective against human glaucoma. Potential protective effects of radiation have been considered for a long time (72-75). Recently, radiation $(0.65 \mathrm{~Gy})$ was shown to protect against retinal degeneration in 2 mouse models (76). Since the protection in that study diminished with time, and the dose was well below the protective dose we demonstrate here, the protective mechanisms are likely to be different from those studied here.

The control of transendothelial migration is very complex and highly context dependent. Therefore, it may not be straightforward to develop highly effective nonradiation-based therapies to lessen glaucoma by preventing monocyte entry into the ONH. Steroids are known to reduce transendothelial migration. In some individuals, steroid use can actually lead to glaucoma (77). This appears contradictory to our findings, but IOP levels are often very high in steroid glaucoma, and this very severe insult may overwhelm any protective antiinflammatory effect. Alternatively, endothelial cells in different vascular beds may behave differently in response to steroids, with possibly a smaller effect on transendothelial migration in the ONH compared with that in other tissues. Statins are also known to reduce transendothelial migration. Interestingly, some studies have shown that long-term statin use appears to be associated with a reduced risk of open-angle glaucoma $(78,79)$. However, given both the robust and long-term efficacy of a single dose of $x$ ray radiation in preventing cellular entry into the optic nerve and retina, it will be important to further evaluate the use of x-rays for preventing glaucoma and other inflammatory conditions.

\section{Methods}

Mouse strain, breeding, and husbandry. Mice were housed in a 14-hourlight/10-hour-dark cycle, under previously described conditions (80). For gene expression profiling, all mice were female and, to minimize environmental differences, were housed under the same conditions in same animal facility. Our DBA/2J mouse colony is routinely crossed with DBA/2J mice from The Jackson Laboratory production facility to prevent genetic drift. The D2-Gpnmb ${ }^{+}$strain (DBA/2J-Gpnmb ${ }^{+} / \mathrm{Sj}[\mathrm{D} 2-\mathrm{Gp}]$ ) was used as a control strain and rarely if ever develops glaucoma in our colony (81). Details for DBA/2J.Thy1(CFP) have been described previously (4).

$\gamma$-Radiation treatment. A lethal dose (10 Gy) of $\gamma$-radiation plus bone marrow reconstitution was administered as described previously (16). Briefly, mice were positioned on a slowly rotating platform to ensure uniform application. Radiation was applied from a ${ }^{137}$ Cesium source in 2 equal doses of 5 Gy spaced 3-4 hours apart. Shortly after the second dose, mice received $200 \mu \mathrm{l}$ intravenous injections containing $5 \times 10^{6}$ bone marrow cells (16). For sublethal doses, mice were exposed to radiation as described for lethal doses but for a shorter periods of time. No bone marrow reconstitution was necessary. For head-only and body-only irradiation, mice were placed individually into a conical tube and placed inside a cylinder of lead shielding with 8-cm thick walls, so that either only the head or only the body and neck were exposed. The shielding system was refined with testing to ensure that, for head-only radiation, the body received no measurable dose of radiation. Likewise, for body-only radiation, the head received no measurable dose of radiation.

$X$-ray radiation treatment. An X-ray machine was specially built to deliver defined doses of $x$-rays to only the eye region. The machine was designed to produce a $3-\mathrm{mm}$ diameter beam and calibrated for accurate dosing of $\mathrm{x}$-rays to the back of the eye at the retina and ONH. Mice were initially swaddled to keep them calm and motionless. Later, when it was determined that anesthesia had no affect on the protection, mice were anesthetized with isoflurane. Mice were placed in a restraining device and positioned $1-\mathrm{mm}$ away from the end of the $\mathrm{x}$-ray delivery tube. Eyes were exposed to defined doses of radiation by varying the time of exposure (e.g., 4 minutes for $7.2 \mathrm{~Gy}$ ). To ensure that the bony orbit was effective at blocking $\mathrm{x}$-rays from penetrating deeper, the cranial skull and a portion of brain from sacrificed mice were removed. X-ray film was then placed directly behind the orbit, and the eye was exposed to different doses of radiation.

Analysis of glaucomatous damage. Intracranial portions of optic nerves were processed and analyzed as previously described (16, 82-84). Briefly, optic nerves were fixed in situ for 48 hours, dissected free, processed, and embedded in plastic. One-micron-thick cross sections of optic nerve from behind the orbit were cut and stained with paraphenylenediamine (PPD). PPD darkly stains the myelin sheaths and axoplasm of sick or dying axons but not healthy axons (85). The 3 clearly distinguishable stages of damage were as follows. (a) In the no or early (NOE) stage, less than $5 \%$ axons were damaged. This amount of damage was seen in age-matched mice of various strains that do not develop glaucoma. It is called NOE, as some of these eyes were undergoing early molecular stages of disease, but they were not distinguishable from eyes with no glaucoma by conventional morphologic analyses of axon number and nerve damage. (b) In the moderate (MOD) stage, there are many damaged axons throughout the nerve, with an average of $30 \%$ axon loss. (c) In the severe (SEV) stage, there was very substantial axon loss (>50\% lost) and damage.

Clinical examination, IOP measurements, axon counts, and RGC soma assessment. DBA/2J mice develop a disease of the iris that leads to IOP elevation and glaucoma. Clinical examinations were performed as previously described (86). The clinical examinations included evaluation of the lens for cataracts. For clinical examinations, at least 20 and typically more than 40 eyes from each genotype or treatment group were examined at $6,8,10$, and 12 months of age. We have studied hundreds of animals that were aged for more than year after radiation treatment and detected no higher prevalence of cataract in $\gamma$-radiation-treated eyes compared with that in untreated eyes. The prevalence of readily detectable cataracts was no greater in 7.2 Gy x-raytreated eyes compared with that in nonirradiated DBA/2J mice, but very mild cataracts were present in a number of these eyes. No additional ocular morbidities (such as tumors) were detected during clinical examinations. Also, during this study, hundreds of radiation-treated eyes were dissected and assessed by light, fluorescent, confocal, and electron microscopy, and no tumors were observed. Finally, we have aged and assessed irradiated (7.2 Gy eye-only x-ray radiation) and nonirradiated C57BL/6J mice $(n=20$ each group) that are heterozygous for a mutation in the $\operatorname{Tr} p 53$ gene that predisposes mice to tumor formation. We did not observed a higher incidence in ocular tumor formation (assessed by detailed clinical examinations) in irradiated mice compared with that in nonirradiated mice.

IOP measurements were performed as described previously $(87,88)$. IOP elevation was confirmed in all cohorts of mice used in this study. Although IOP was not measured in every mouse, many mice in each protected cohort were confirmed to have high IOP. Mice were aged and analyzed with no specific selection criteria for inclusion.

Axon and RGC layer soma counts were preformed as described previously (4). Two-tailed Student $t$ tests were performed for statistical significance. $P<0.05$ was considered significant. 
Anterograde axon transport. Ten-month-old mice were injected with 1-2 $\mu \mathrm{l}$ $(1 \mathrm{mg} / \mathrm{ml})$ Alexa Fluor 594-Cholera Toxin subunit B conjugate (Invitrogen) into the vitreous, using a Hamilton syringe with a 35 -gauge needle. After 48 to 72 hours, they were anesthetized and euthanized via 4\% PFA cardiac perfusion. Brains were submersion fixed for 24 hours after perfusion, cryoprotected in $30 \%$ sucrose overnight, OCT cryoembedded, and sectioned at $50 \mu \mathrm{m}$. Alexa Fluor 594 was visualized using an SP5 confocal microscope (Leica).

Assessing RGC axon damage in D2.Thy1-CFP and irradiated D2.Thy1-CFP mice. Dystrophic neurites and regions of focal damage (axonal swellings) were assessed as described previously (4). Briefly, eyes were fixed, and retinas with ONH still attached were flat mounted onto a slide, inner retina uppermost, and coverslipped. An SP5 confocal microscope (Leica) was used to visualize $\mathrm{CFP}$ fluorescence. Location within the $\mathrm{ONH}$ was determined using the position of the central retinal vessels as landmarks as described previously (4).

PERG. Pattern electroretinography was measured as previously reported $(22,23,89)$. Mice from our colony were shipped in a "masked" fashion to the University of Miami, Miami, Florida, USA. Full-field, flash ERG was used as a control on all mice (an outer retina signal that is not affected by RGCs), and it did not differ significantly between control and glaucomatous DBA/2J mice.

Genome-wide gene expression profiling. Tissue harvesting and RNA isolation and processing for microarrays was as described previously (26). Briefly, the ONH was excised using a sharpened 1-mm glass capillary centered over the optic nerve. The punched out nerve head was immediately placed in RNAlater (Ambion). This tissue is substantially enriched for the glial lamina (26). For RNA preparation, total RNA was isolated using the RNeasy Micro Kit (Qiagen). For RNA labeling and microarray processing, biotin-labeled cDNA was synthesized separately from 20 ng of each total RNA sample per the manufacturer's protocols (Ovation Biotin System, NuGEN). $2.5 \mu \mathrm{g}$ of each biotin-labeled and fragmented cDNA sample was then hybridized onto Mouse Genome 430 v2.0 GeneChip arrays (Affymetrix). Staining and washing after hybridization were performed according to the manufacturer's protocols using the Fluidics Station 450 instrument (Affymetrix). Finally, the arrays were scanned with a GeneChip Scanner 3000 laser confocal slide scanner. The images were quantified using GeneChip Operating Software v1.2. Probe level data were imported into the R statistical software environment, and expression values for each probe set on the array were summarized using the robust multichip average method with quantile normalization in the R/affy package $(90,91)$.

All mice were aged and harvested over the same time period. All 80 samples were processed at the same time, and quantile normalization of all of the arrays was performed. All data are available in the GEO database (NCBI, accession no. GSE26299; http://www.ncbi.nlm.nih.gov/geo/). Results from a previously analyzed subset of samples were previously published (26). A subset of 50 of these samples was analyzed for the first time during the current study. These $50 \mathrm{ONH}$ samples (10 D2-Gpnmb ${ }^{+}$control samples, $20 \mathrm{NOE}$ DBA/2J samples, and 10 radiation-treated DBA2J samples all at 8.5 months of age and 10 radiation-treated DBA/2J samples at 10.5 months of age) were combined with the 30 samples described previously (10 D2-Gpnmb ${ }^{+}$ samples and $20 \mathrm{NOE}$ DBA/2J samples, both at 10.5 months of age; ref. 26), and all 80 were analyzed together for the current study.

Defining early stages of disease using hierarchical clustering. By comparing controls without glaucoma to all standard DBA/2J eyes, a set of disease-associated probe sets was identified as previously reported (26). These probe sets were used to perform hierarchical clustering of the $80 \mathrm{ONH}$ samples (using JMP Statistical Discovery v7.0 software). Using the average distance metric, hierarchical clustering started with each eye as its own cluster. At each step, the clustering algorithm calculated the distance between each cluster and combined the 2 clusters that were closest together. This continued until all the points were in one final cluster or dendrogram. To identify samples that clustered into specific glaucoma stages, a threshold or cutoff was applied as described previously (26). The threshold was very similar to that reported, and the samples that clustered in stage 2 in that study also clustered together in the current study (along with a few additional samples). Thus, stage 2 was recreated in the current study. In contrast and due to the study of more eyes at very early stages of glaucoma, the samples that were in stage 1 in our previous study clustered into 3 new molecular stages that contained many of the newly analyzed samples. The new clusters represent newly identified glaucoma stages (referred to as stages $1 \mathrm{a}, 1 \mathrm{~b}$, and $1 \mathrm{c}$ for consistency), each of which contained at least 5 samples. Hierarchical clustering identified a group of 10 radiation-treated eyes (Rad-D2 eyes) that were most similar to untreated eyes in stage $1 \mathrm{~b}$. D2-Gpnmb ${ }^{+}$control eyes clustered into 2 groups (D2-Gp1 and D2-Gp2). This indicates that, despite no visibly detectable differences between samples in either group, molecular differences are sufficient to distinguish D2-Gpnmb $b^{+}$samples. Despite these differences, D2-Gp1 and D2-Gp2 are still more similar to each other than any glaucoma stage. However, D2-Gp1 was more distant from the glaucoma stages than D2-Gp2 and was therefore used as the control group (D2-Gp) in this study (Figure 3). Comparing to D2-Gp2 provides similar overall results.

Identification of DE genes and bioinformatics analysis. Statistical analysis for differential gene expression between any 2 groups was assessed by genespecific fixed-effect ANOVA methods using the R/manova package (90). All $P$ values were calculated by permuting model residuals 1,000 times. To address the problem of multiple testing, the permutated $P$ values were adjusted using the false discovery rate (FDR) method (92) implemented in $\mathrm{R} / q$ value. The resulting $q$ values estimated the proportion of false positives in the list of DE genes and were used to select suitable candidate gene lists for subsequent analysis. Unless otherwise noted, statistical significance was determined at $q<0.05$ (FDR $<5 \%$ ). Probe sets were annotated using Netaffx software (www.affymetrix.com/analysis/netaffx).

To identify pathways overrepresented in DE gene lists, the Database for Annotation, Visualization and Integrated Discovery (ref. 30) was used. Pathways showing upregulated and downregulated genes were constructed using Kyoto Encyclopedia of genes and genomes (KEGG) pathways (29) and Gene ontology terms (93).

$\mathrm{ONH} /$ retina dissociation for flow cytometry. Eyes were enucleated from the mouse immediately after euthanization. Retinas and optic nerves were washed and dissected freely in $1 \times$ PBS. Retinas were placed in $50 \mu 10.25 \%$ trypsin-EDTA and incubated at $37^{\circ} \mathrm{C}$ for 5 minutes, triturated, and returned to $37^{\circ} \mathrm{C}$ for 1 minute. The solution was further trypsinized by $100 \mu 10.25 \%$ trypsin-EDTA, and remaining cell clumps were removed. Trypsinization was halted by soybean trypsinase. Optic nerves were placed in $50 \mu 15 \mathrm{mg} / \mathrm{ml}$ Dispase HBSS (STEMCELL Technologies) at $37^{\circ} \mathrm{C}$, with shaking for 1 hour. The digestion was stopped with DMEM/high glucose with FBS.

Cells were stained with the various antibodies for 45 minutes at $4{ }^{\circ} \mathrm{C}$. The cells were centrifuged at $300 \mathrm{~g}$ to $500 \mathrm{~g}$ for 5 minutes and then washed with $1 \times$ PBS containing $5 \mathrm{mM}$ EDTA, $2 \% \mathrm{FBS}$, and $0.02 \% \mathrm{NaN}_{3}$. Samples were centrifuged and washed one more time. Antibodies were titrated prior to use, and optimal concentrations were used. Compensation controls used rat/hamster BD CompBeads (BD Biosciences). Cells were sorted on a BD Biosciences LSR II SORP, with 4 lasers at the following wavelengths: $355 \mathrm{~nm}$, $405 \mathrm{~nm}, 488 \mathrm{~nm}$, and $633 \mathrm{~nm}$. Acquisition was in FACSDiVa software (BD Biosciences) and analyzed with Flowjo (TreeStar Inc.). Antibodies used were CD106 (clone 429, BD Biosciences), F4/80 PE (Cedarlane), CD11b (clone M1/70, Biolegend), CD34 (clone RAM34, eBioscience), CD11c (clone N418, Biolegend), Gr-1 (clone RB6-8C5, Biolegend), CD45.2 (clone 1042.1 grown, purified, and labeled with Pacific Orange Protein Labeling Kit, Invitrogen).

Immunofluorescence. DBA/2J.Thy1-CFP mice were used to visualize RGCs (4). Immunofluorescence to visualize IBA1, MBP, CD68, EDN2, and NFL was performed as follows. Eyes were fixed overnight at $4^{\circ} \mathrm{C}$ in $4 \%$ PFA. Ret- 
inal whole mounts were processed as described previously (26). Primary antibodies used were anti-EDN2 (Santa Cruz Biotechnology Inc., 1:100), anti-MBP (Chemicon, 1:500), anti-CD68 (Abdserotec, 1:500), anti-IBA1 (WAKO, 1:200), MECA-79 (Santa Cruz Biotechnology Inc., 1:50), and antiNFL (DakoCytomation, 1:1,000). Fluorescence was visualized using an SP5 confocal microscope (Leica). For ONH sections, eyes were fixed overnight at $4{ }^{\circ} \mathrm{C}$ in $4 \% \mathrm{PFA}$ and processed as described previously (4).

For all immunohistochemistry, at least 3 sections $(\mathrm{ONH})$ or whole retinas from 6 to 10 untreated and radiation-treated DBA/2J eyes and at least 5 to $10 \mathrm{D} 2-\mathrm{Gpnmb} b^{+}$control eyes were assessed.

Monocyte counting. For the ONH, the Leica DMRE microscope system with MetaMorph software version 6.3r7 was used to image the optic nerve under a $10 \times$ objective. The optic nerve image was uploaded into Fiji software (http://fiji.sc/wiki/index.php/Fiji). The ONH region counted was defined as being from the top of the $\mathrm{ONH}$ to $50 \mu \mathrm{m}$ past the myelin transition zone (MTZ). The software calculated the area of the region for each section. A microglia was defined as a DAPI-stained nuclei that was closely overlapping or touching a region of IBA1 staining (Figure 6E). Microglia in each region were counted, and the density of microglia (number of microglia per area of region) was calculated.

For the retina, the Zeiss Axio Observer Z.1 microscope was used to image the entire surface of the inner retina, with the $20 \times$ objective, creating a tiled image. The tiled image was uploaded into Fiji. The total area of the retina was calculated. IBA $1^{+} \mathrm{CD} 68^{+}$cells for each retina were counted and characterized as either ramified or round.

Cell tracking with CFDA. Splenocytes were labeled using 6-CFDA (Invitrogen) via intrasplenic injection. Lyophilized 6-CFDA was brought to room temperature and solubilized in sterile $1 \times$ PBS under aseptic conditions to reach $2 \% \mathrm{w} / \mathrm{v}$. Mice were anesthetized using isoflurane. A vertical incision of one-third of an inch was made through the skin above the spleen to expose the peritoneal musculature. A second incision was made to the peritoneal musculature under the opening in the skin. Using forceps, the spleen was gently exteriorized through the 2 incisions. Using a cotton swab to stabilize the spleen, the spleen was injected directly with $100 \mu \mathrm{l}$ of $2 \%$ 6-CFDA with a 30-gauge needle. The spleen was carefully replaced inside the peritoneal wall, and the peritoneal cavity was sutured using 5-0 coated vicryl in an interrupted stitch pattern. The outer incision was closed with 1 wound clip. The mouse was wrapped loosely in sterile gauze and placed under a warming lamp until fully recovered from anesthesia. 72 hours later, mice were sacrificed, and eyes were harvested and fixed in 4\% PFA for 1 hour. Optic nerves and retinas were removed from the eyes and placed on a glass slide. CFDA was visualized using an SP5 confocal microscope (Leica).

Vessel measurements, EDN2 injections, and optic nerve crush. Retinas were fixed in $4 \%$ PFA overnight, placed flat on a slide, and coverslipped. Major arterioles were measured from 24 imaged fields that were evenly distributed around the retina, $1 \mathrm{~mm}$ out from the $\mathrm{ONH}$. Images were taken using differential interference contrast on an SP5 confocal microscope (Leica). For each vessel, the diameter of both the lumen and the outer vessel wall (taken from widest part of the vessel present in the field) was calculated as the average of 3 measurements. The ratio of the lumen areas to the total vessel area was calculated (area $=\pi r^{2}$, where $r=$ radius). Bosentan, an endothelin receptor antagonist, was provided by Actelion Pharmaceuticals. Bosentan was incorporated into standard mouse chow $(100 \mathrm{mg} / \mathrm{kg})$.

For intravitreal injections of EDN2, $500 \mathrm{mM}$ EDN2 peptide (dissolved in $2 \mu \mathrm{l}$ of sterile $1 \times$ PBS, Invitrogen) was injected into the vitreous chamber using a Hamilton syringe with a 35 -gauge needle. $1 \times$ PBS (vehicle) was injected as a control. Mice were 10-months-old at the time of injection and after 4 weeks, optic nerves were harvest and assessed for optic nerve damage (see Analysis of glaucomatous damage).

Controlled optic nerve crush was performed as described previously $(83,94)$

Statistics. In figures, all data are presented as mean \pm SEM. For IOP data, 2 -tailed Student's $t$ tests were performed for statistical significance. For radiation experiments, differences in optic nerve damage distributions among groups were compared using $\chi^{2}$ tests. Unless specifically stated in the text, $P<0.05$ was considered significant.

Study approval. Experiments were conducted in accordance with the Association for Research in Vision and Ophthalmology statement on the use of animals in ophthalmic research. The Jackson Laboratory Animal Care and Use Committee approved all of the experiments in this study.

\section{Acknowledgments}

We are grateful to Evgeny Sozontov, Emil Strumban, and the late George Gutman for designing and building the $\mathrm{x}$-ray machine for ocular treatment. We thank Cammie Phalan for radiation treatments, Amy Bell for IOP measurements, Adrienne Mehalow for tissue harvesting and technical support, and K. Saidas Nair, Krish Kizhatil, Mimi de Vries, Jeffrey Marchant, and David Serreze for advice and critical comments. We thank Steven Rosen (UCSF) for insightful discussion. We also acknowledge Scientific Services at The Jackson Laboratory, particularly Gene Expression, Histology, and the Computational Sciences. Significant support was provided by The Partridge Foundation and National Eye Institute (NEI) EY011721 (to S.W.M. John). Other funding was provided by NEI EY018606 (to R.T. Libby), NIH T32 NS51112-05 (to I. Soto), The Barbara and Joseph Cohen Foundation, donors of National Glaucoma Research - a program of the American Health Assistance Foundation (to G.R. Howell), and a Research to Prevent Blindness Career Development Award (to R.T. Libby). S.W.M. John is a Howard Hughes Medical Institute Investigator.

Received for publication September 22, 2011, and accepted in revised form January 25, 2012.

Address correspondence to: Simon W.M. John, Howard Hughes Medical Institute, The Jackson Laboratory, 600 Main St., Bar Harbor, Maine 04609, USA. Phone: 207.288.6496; Fax: 207.288.6078; E-mail: simon.john@jax.org.
1. Whitmore AV, Libby RT, John SW. Glaucoma: thinking in new ways-a role for autonomous axonal self-destruction and other compartmentalised processes? Prog Retin Eye Res. 2005;24(6):639-662.

2. Quigley HA, Broman AT. The number of people with glaucoma worldwide in 2010 and 2020. BrJ Ophthalmol. 2006;90(3):262-267.

3. Quigley HA, Addicks EM. Regional differences in the structure of the lamina cribrosa and their relation to glaucomatous optic nerve damage. Arch Ophthalmol. 1981;99(1):137-143.

4. Howell GR, et al. Axons of retinal ganglion cells are insulted in the optic nerve early in DBA/2J glau- coma. J Cell Biol. 2007;179(7):1523-1537.

5. May CA, Lütjen-Drecoll E. Morphology of the murine optic nerve. Invest Ophthalmol Vis Sci. 2002; 43(7):2206-2212.

6. Schlamp CL, Li Y, Dietz JA, Janssen KT, Nickells RW. Progressive ganglion cell loss and optic nerve degeneration in DBA/2J mice is variable and asymmetric. BMC Neurosci. 2006;7:66.

7. Anderson DR. Ultrastructure of human and monkey lamina cribrosa and optic nerve head. Arch Ophthalmol. 1969;82(6):800-814.

8. Anderson DR, Hendrickson AE. Failure of increased intracranial pressure to affect rapid axonal trans- port at the optic nerve head. Invest Ophthalmol Vis Sci. 1977;16(5):423-426.

9. Chidlow G, Ebneter A, Wood JP, Casson RJ. The optic nerve head is the site of axonal transport disruption, axonal cytoskeleton damage and putative axonal regeneration failure in a rat model of glaucoma. Acta Neuropathol. 2011;121(6):737-751.

10. Morrison JC, Dorman-Pease ME, Dunkelberger GR, Quigley HA. Optic nerve head extracellular matrix in primary optic atrophy and experimental glaucoma. Arch Ophthalmol. 1990;108(7):1020-1024.

11. Quigley HA, Addicks EM, Green WR, Maumenee AE. Optic nerve damage in human glaucoma. II. 
The site of injury and susceptibility to damage. Arch Ophthalmol. 1981;99(4):635-649.

12. Quigley HA, Hohman RM, Addicks EM. Quantitative study of optic nerve head capillaries in experimental optic disk pallor. Am J Ophthalmol. 1982; 93(6):689-699.

13. Radius RL, Anderson DR. Morphology of axonal transport abnormalities in primate eyes. $\mathrm{Br} J$ Ophthalmol. 1981;65(11):767-777.

14. Jakobs TC, Libby RT, Ben Y, John SW, Masland RH. Retinal ganglion cell degeneration is topological but not cell type specific in DBA/2J mice. J Cell Biol. 2005;171(2):313-325.

15. May CA, Mittag T. Optic nerve degeneration in the $\mathrm{DBA} / 2 \mathrm{NNia}$ mouse: is the lamina cribrosa important in the development of glaucomatous optic neuropathy? Acta Neuropathol. 2006;111(2):158-167.

16. Anderson MG, Libby RT, Gould DB, Smith RS, John SW. High-dose radiation with bone marrow transfer prevents neurodegeneration in an inherited glaucoma. Proc Natl Acad Sci U S A. 2005; 102(12):4566-4571.

17. Yamada M, Wong FL, Fujiwara S, Akahoshi M, Suzuki G. Noncancer disease incidence in atomic bomb survivors, 1958-1998. Radiat Res. 2004; 161(6):622-632.

18. Otani A, et al. Rescue of retinal degeneration by intravitreally injected adult bone marrow-derived lineage-negative hematopoietic stem cells. J Clin Invest. 2004;114(6):765-774.

19. Crish SD, Sappington RM, Inman DM, Horner PJ, Calkins DJ. Distal axonopathy with structural persistence in glaucomatous neurodegeneration. Proc Natl Acad Sci U S A. 2010;107(11):5196-5201.

20. Quigley HA, Addicks EM. Chronic experimental glaucoma in primates. II. Effect of extended intraocular pressure elevation on optic nerve head and axonal transport. Invest Ophthalmol Vis Sci. 1980; 19(2):137-152.

21. Anderson DR, Hendrickson A. Effect of intraocular pressure on rapid axoplasmic transport in monkey optic nerve. Invest Ophthalmol. 1974;13(10):771-783.

22. Porciatti V, Saleh M, Nagaraju M. The pattern electroretinogram as a tool to monitor progressive retinal ganglion cell dysfunction in the $\mathrm{DBA} / 2 \mathrm{~J}$ mouse model of glaucoma. Invest Ophthalmol Vis Sci. 2007;48(2):745-751.

23. Porciatti V, Chou TH, Feuer WJ. C57BL/6J, DBA/2J, and DBA/2J.Gpnmb mice have different visual signal processing in the inner retina. Mol Vis. 2010;16:2939-2947.

24. Gutman G, Strumban E, Sozontov E, Jenrow K. Xray scalpel - a new device for targeted $\mathrm{x}$-ray brachytherapy and stereotactic radiosurgery. Phys Med Biol. 2007;52(6):1757-1770.

25. Quigley HA, Hohman RM, Addicks EM, Massof RW, Green WR. Morphologic changes in the lamina cribrosa correlated with neural loss in open-angle glaucoma. Am J Ophthalmol. 1983;95(5):673-691.

26. Howell GR, et al. Molecular clustering identifies complement and endothelin induction as early events in a mouse model of glaucoma. J Clin Invest. 2011; 121(4):1429-1444.

27. Fevre-Montange M, et al. Microarray gene expression profiling in meningiomas: differential expression according to grade or histopathological subtype. Int J Oncol. 2009;35(6):1395-1407.

28. Knosel T, et al. Immunoprofiles of 11 biomarkers using tissue microarrays identify prognostic subgroups in colorectal cancer. Neoplasia. 2005; 7(8):741-747.

29. Kanehisa M, et al. KEGG for linking genomes to life and the environment. Nucleic Acids Res. 2008; 36(database issue):D480-D484.

30. Sherman BT, et al. DAVID Knowledgebase: a genecentered database integrating heterogeneous gene annotation resources to facilitate high-throughput gene functional analysis. BMC Bioinformatics.
2007;8:426.

31. Uchimura K, Rosen SD. Sulfated L-selectin ligands as a therapeutic target in chronic inflammation. Trends Immunol. 2006;27(12):559-565.

32. Lowe JB. Glycosylation in the control of selectin counter-receptor structure and function. Immunol Rev. 2002;186:19-36.

33. Perry VH, Andersson PB, Gordon S. Macrophages and inflammation in the central nervous system. Trends Neurosci. 1993;16(7):268-273.

34. Perry VH, Nicoll JA, Holmes C. Microglia in neurodegenerative disease. Nat Rev Neurol. 2010; 6(4):193-201.

35. King IL, Dickendesher TL, Segal BM. Circulating Ly-6C+ myeloid precursors migrate to the CNS and play a pathogenic role during autoimmune demyelinating disease. Blood. 2009;113(14):3190-3197.

36. Fischer HG, Reichmann G. Brain dendritic cells and macrophages/microglia in central nervous system inflammation. IImmunol. 2001;166(4):2717-2726.

37. Lehmann U, Heuss ND, McPherson SW, Roehrich H, Gregerson DS. Dendritic cells are early responders to retinal injury. Neurobiol Dis. 2010;40(1):177-184.

38. Mizoguchi A, et al. Dependence of intestinal granuloma formation on unique myeloid DC-like cells. J Clin Invest. 2007;117(3):605-615.

39. Reichmann G, Schroeter M, Jander S, Fischer HG. Dendritic cells and dendritic-like microglia in focal cortical ischemia of the mouse brain.J Neuroimmunol. 2002;129(1-2):125-132.

40. Krishnamoorthy RR, Rao VR, Dauphin R, Prasanna G, Johnson C, Yorio T. Role of the ETB receptor in retinal ganglion cell death in glaucoma. Can J Physiol Pharmacol. 2008;86(6):380-393.

41. Noske W, Hensen J, Wiederholt M. Endothelin-like immunoreactivity in aqueous humor of patients with primary open-angle glaucoma and cataract. Graefes Arch Clin Exp Ophthalmol. 1997;235(9):551-552.

42. Panagis L, Zhao X, Ge Y, Ren L, Mittag TW, Danias J. Gene expression changes in areas of focal loss of retinal ganglion cells (RGC) in the retina of DBA/2J mice. Invest Ophthalmol Vis Sci. 2010;51(4):2024-2034

43. Tezel G, Kass MA, Kolker AE, Becker B, Wax MB. Plasma and aqueous humor endothelin levels in primary open-angle glaucoma. J Glaucoma. 1997; 6(2):83-89.

44. Nakazawa $T$, et al. Tumor necrosis factor-alpha mediates oligodendrocyte death and delayed retinal ganglion cell loss in a mouse model of glaucoma. J Neurosci. 2006;26(49):12633-12641.

45. Jiang B, et al. Neuroinflammation in advanced canine glaucoma. Mol Vis. 2010;16:2092-2108.

46. Johnson EC, et al. Cell proliferation and interleukin-6-type cytokine signaling are implicated by gene expression responses in early optic nerve head injury in rat glaucoma. Invest Ophthalmol Vis Sci. 2011; 52(1):504-518.

47. Johnson EC, Jia L, Cepurna WO, Doser TA, Morrison JC. Global changes in optic nerve head gene expression after exposure to elevated intraocular pressure in a rat glaucoma model. Invest Ophthalmol Vis Sci. 2007;48(7):3161-3177.

48. Kompass KS, Agapova OA, Li W, Kaufman PL, Rasmussen CA, Hernandez MR. Bioinformatic and statistical analysis of the optic nerve head in a primate model of ocular hypertension. BMC Neurosci. 2008;9:93.

49. Steele MR, Inman DM, Calkins DJ, Horner PJ, Vetter ML. Microarray analysis of retinal gene expression in the DBA/2J model of glaucoma. Invest Ophthalmol Vis Sci. 2006;47(3):977-985.

50 . Yang $Z$, et al. Changes in gene expression in experimental glaucoma and optic nerve transection: the equilibrium between protective and detrimental mechanisms. Invest Ophthalmol Vis Sci. 2007; 48(12):5539-5548.

51. Sofroniew MV. Molecular dissection of reactive astrogliosis and glial scar formation. Trends Neurosci.
2009;32(12):638-647.

52. Farina C, Aloisi F, Meinl E. Astrocytes are active players in cerebral innate immunity. Trends Immunol. 2007;28(3):138-145

53. Leon S, Yin Y, Nguyen J, Irwin N, Benowitz LI. Lens injury stimulates axon regeneration in the mature rat optic nerve. J Neurosci. 2000;20(12):4615-4626.

54. Yin Y, et al. Oncomodulin is a macrophage-derived signal for axon regeneration in retinal ganglion cells. Nat Neurosci. 2006;9(6):843-852.

55. Wax MB, Tezel G. Immunoregulation of retinal ganglion cell fate in glaucoma. Exp Eye Res. 2009; 88(4):825-830.

56. Schwartz M, London A. Immune maintenance in glaucoma: boosting the body's own neuroprotective potential. J Ocul Biol Dis Infor. 2009;2(2):73-77.

57. Ebneter A, Casson RJ, Wood JP, Chidlow G. Microglial activation in the visual pathway in experimental glaucoma: spatiotemporal characterization and correlation with axonal injury. Invest Ophthalmol Vis Sci. 2010;51(12):6448-6460

58. Son JL, et al. Glaucomatous optic nerve injury involves early astrocyte reactivity and late oligodendrocyte loss. Glia. 2010;58(7):780-789.

59. Rosen SD. Ligands for L-selectin: homing, inflammation, and beyond. Annu Rev Immunol. 2004; 22:129-156.

60. Wang N, Chintala SK, Fini ME, Schuman JS. Activation of a tissue-specific stress response in the aqueous outflow pathway of the eye defines the glaucoma disease phenotype. Nat Med. 2001; 7(3):304-309.

61. Krejci J, et al. Epigenetics of multiple myeloma after treatment with cytostatics and gamma radiation. Leuk Res. 2009;33(11):1490-1498.

62. Hwang ST, et al. GlyCAM-1, a physiologic ligand for L-selectin, activates beta 2 integrins on naive peripheral lymphocytes. J Exp Med. 1996; 184(4):1343-1348.

63. Kansas GS. Selectins and their ligands: current concepts and controversies. Blood. 1996; 88(9):3259-3287.

64. Hoke D, et al. Selective modulation of the expression of L-selectin ligands by an immune response. Curr Biol. 1995;5(6):670-678.

65. Johnson EC, Morrison JC. Friend or foe? Resolving the impact of glial responses in glaucoma. J Glaucoma. 2009;18(5):341-353.

66. Flammer J, Mozaffarieh M. What is the present pathogenetic concept of glaucomatous optic neuropathy? Surv Ophthalmol. 2007;52 suppl 2:S162-S173.

67. Downs JC, Roberts MD, Burgoyne CF. Mechanical environment of the optic nerve head in glaucoma. Optom Vis Sci. 2008;85(6):425-435.

68. Zhong L, et al. Erythropoietin promotes survival of retinal ganglion cells in DBA/2J glaucoma mice. Invest Ophthalmol Vis Sci. 2007;48(3):1212-1218.

69. Lambert WS, Ruiz L, Crish SD, Wheeler LA, Calkins DJ. Brimonidine prevents axonal and somatic degeneration of retinal ganglion cell neurons. $\mathrm{Mol}$ Neurodegener. 2011;6(1):4

70. Almasieh M, Zhou Y, Kelly ME, Casanova C, Di Polo A. Structural and functional neuroprotection in glaucoma: role of galantamine-mediated activation of muscarinic acetylcholine receptors. Cell Death Dis. 2010;1:e27.

71. Finger PT. Radiation therapy for orbital tumors: concepts, current use, and ophthalmic radiation side effects. Survey of ophthalmology. 2009;54(5):545-568.

72 . Hively W. Is radiation good for you? Or dioxin? Or arsenic? Discover. 2002;12:74-80.

73. Kipnis J, et al. Low-dose gamma-irradiation promotes survival of injured neurons in the central nervous system via homeostasis-driven proliferation of T cells. Eur J Neurosci. 2004;19(5):1191-1198.

74. Sagan LA. On radiation, paradigms, and hormesis. Science. 1989;245(4918):574, 621

75 . Wolff S. Are radiation-induced effects hormetic? 
Science. 1989;245(4918):575, 621.

76. Otani A, Kojima H, Guo C, Oishi A, Yoshimura N. Low-dose-rate, low-dose irradiation delays neurodegeneration in a model of retinitis pigmentosa. Am J Pathol. 2012;180(1):328-336.

77. Clark AF, Wordinger RJ. The role of steroids in outflow resistance. Exp Eye Res. 2009;88(4):752-759.

78. Marcus MW, et al. Cholesterol-lowering drugs and incident open-angle glaucoma: a population-based cohort study. PLoS One 2012;7(1):e29724.

79. McGwin G Jr, McNeal S, Owsley C, Girkin C, Epstein D, Lee PP. Statins and other cholesterollowering medications and the presence of glaucoma. Arch Ophthalmol. 2004;122(6):822-826.

80. Smith RS, et al. Haploinsufficiency of the transcription factors FOXC1 and FOXC2 results in aberrant ocular development. Hum Mol Genet. 2000; 9(7):1021-1032.

81. Howell GR, et al. Absence of glaucoma in DBA/2J mice homozygous for wild-type versions of Gpnmb and Tyrp1. BMC Genet. 2007;8:45.

82. Anderson MG, et al. Genetic context determines susceptibility to intraocular pressure elevation in a mouse pigmentary glaucoma. BMC Biol. 2006; 4:20.

83. Libby RT, et al. Susceptibility to neurodegeneration in a glaucoma is modified by Bax gene dosage. PLoS Genet. 2005;1(1):17-26.

84. Libby RT, et al. Inherited glaucoma in DBA/2J mice: pertinent disease features for studying the neurodegeneration. Vis Neurosci. 2005;22(5):637-648.

85. Sadun AA, Smith LE, Kenyon KR. Paraphenylenediamine: a new method for tracing human visual pathways. J Neuropathol Exp Neurol. 1983; 42(2):200-206.

86. Anderson MG, et al. Mutations in genes encoding melanosomal proteins cause pigmentary glaucoma in DBA/2J mice. Nat Genet. 2002;30(1):81-85.

87. John SW, Hagaman JR, MacTaggart TE, Peng L, Smithes O. Intraocular pressure in inbred mouse strains. Invest Ophthalmol Vis Sci. 1997;38(1):249-253.

88. Savinova OV, et al. Intraocular pressure in genetically distinct mice: an update and strain survey. BMC Genet. 2001;2:12.
89. Porciatti V. The mouse pattern electroretinogram. Doc Ophthalmol. 2007;115(3):145-153.

90. Churchill GA. Using ANOVA to analyze microarray data. Biotechniques. 2004;37(2):173-175.

91. Gautier L, Cope L, Bolstad BM, Irizarry RA. affy-analysis of Affymetrix GeneChip data at the probe level. Bioinformatics. 2004;20(3):307-315.

92. Storey JD, Tibshirani R. Statistical significance for genomewide studies. Proc Natl Acad Sci U S A. 2003;100(16):9440-9445.

93. Gene Ontology Consortium. The Gene Ontology (GO) project in 2006. Nucleic Acids Res. 2006; 34(database issue):D322-D326.

94. Li Y, Schlamp CL, Poulsen KP, Nickells RW. Baxdependent and independent pathways of retinal ganglion cell death induced by different damaging stimuli. Exp Eye Res. 2000;71(2):209-213.

95. Lemstra AW, et al. Microglia activation in sepsis: a case-control study. J Neuroinflammation. 2007;4:4.

96. Roux S, Breu V, Ertel SI, Clozel M. Endothelin antagonism with bosentan: a review of potential applications. J Mol Med (Berl). 1999;77(4):364-376. 